\title{
FORECASTING THE WIND POWER GENERATION IN CHINA BY SEASONAL GREY FORECASTING MODEL BASED ON COLLABORATIVE OPTIMIZATION
}

\author{
Aodi Sui And Wuyong Qian*
}

\begin{abstract}
Renewable energy represented by wind energy plays an increasingly important role in China's national energy system. The accurate prediction of wind power generation is of great significance to China's energy planning and power grid dispatch. However, due to the late development of the wind power industry in China and the lag of power enterprise information, there are little historical data available at present. Therefore, the traditional large sample prediction method is difficult to be applied to the forecasting of wind power generation in China. For this kind of small sample and poor information problem, the grey prediction method can give a good solution. Thus, given the seasonal and long memory characteristics of the seasonal wind power generation, this paper constructs a seasonal discrete grey prediction model based on collaborative optimization. On the one hand, the model is based on moving average filtering algorithm to realize the recognition of seasonal and trend features. On the other hand, based on the optimization of fractional order and initial value, the collaborative optimization of trend and season is realized. To verify the practicability and accuracy of the proposed model, this paper uses the model to predict the quarterly wind power generation of China from 2012Q1 to 2020Q1, and compares the prediction results with the prediction results of the traditional GM $(1,1)$ model, SGM(1,1) model and Holt-Winters model. The results are shown that the proposed model has a strong ability to capture the trend and seasonal fluctuation characteristics of wind power generation. And the long-term forecasts are valid if the existing wind power expansion capacity policy is maintained in the next four years. Based on the forecast of China's wind power generation from 2021Q2 to 2024Q2 in the future, it is predicted that China's wind power generation will reach $239.09 \mathrm{TWh}$ in the future, which will be beneficial to the realization of China's energy-saving and emission reduction targets.
\end{abstract}

Mathematics Subject Classification. 90B99, 62P30.

Received March 8, 2021. Accepted August 29, 2021.

\section{INTRODUCTION}

\subsection{Background and motivation}

In recent years, as a new type of energy, wind power has received widespread attention due to its good environmental benefits. Wind power is regarded as an excellent alternative to traditional fossil energy [6].

Keywords. Discrete grey forecasting model, fractional-order accumulation generation, seasonal adjustment, wind power generation.

School of Business, Jiangnan University, Wuxi, Jiangsu 214122, P.R. China.

*Corresponding author: wuyongqian@jiangnan.edu.cn 


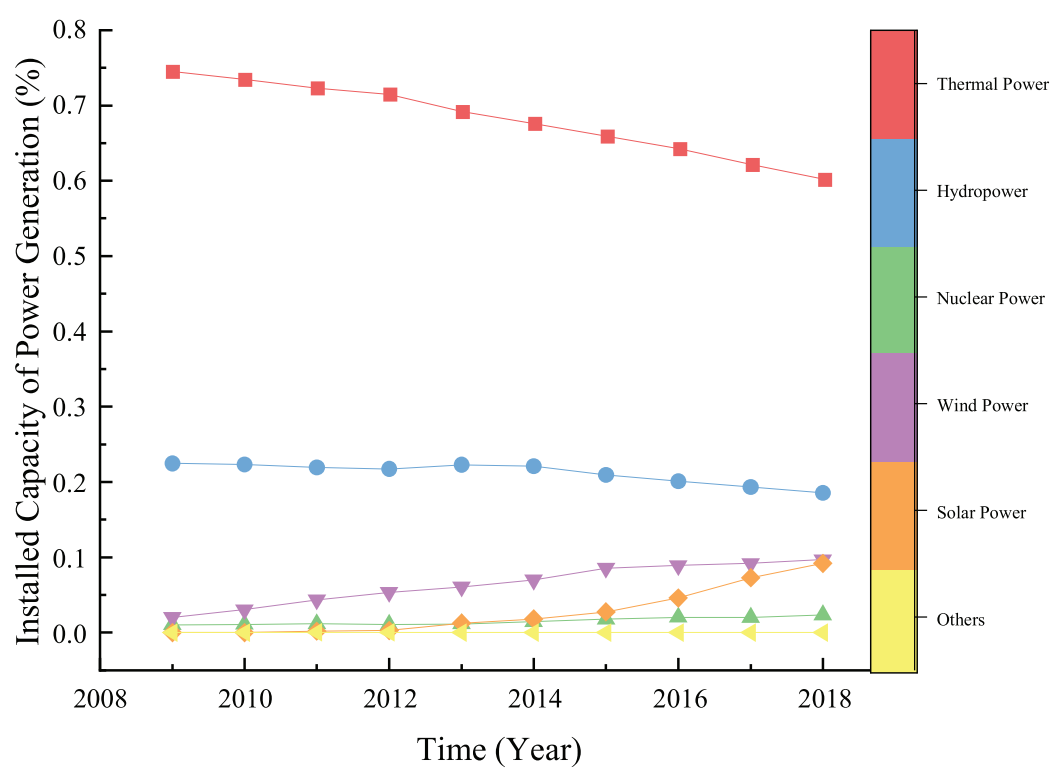

FIGURE 1. Installed capacity of power generation in China.

As shown in Figure 1 (https://data.stats.gov.cn/easyquery.htm?cn=A01), China's installed wind power generation capacity in 2018 was 184.26 million kilowatts, accounting for $10 \%$ of the total installed capacity of generators. In the first eight months of 2018, meanwhile, China's wind power generation growth rate was $20.5 \%$, and wind power generation accounted for $4.75 \%$ of China's total power generation. As a result, wind power has become an important part of China's energy strategy. Although wind power has significant environmental benefits, the intermittent and uncertain characteristics of wind power have limited the development of the wind power industry $[26,29]$. Unexpected changes in wind farm production may increase the operating costs of the power system and pose a potential risk to the reliability of the power supply [12]. Therefore, it is of great significance to accurately forecast wind power generation in China and to clarify the long-term development trend and seasonal characteristics of wind power generation. Accurate forecasting of wind power generation can not only promote the realization of energy-saving and emission reduction targets, reduce power company operating costs, improve energy efficiency, optimize grid design and energy allocation, but also have important implications for energy structure adjustment and industrial structure optimization.

However, due to the short development time of the wind power industry in China and the low degree of informatization of power companies in some regions, so we cannot obtain a large amount of historical data [24]. And the factors that affect wind power generation are complex. Therefore, the characteristics of small sample sizes and poor information of China's wind power generation limit the application of prediction methods based on large sample sizes. Grey system theory is an effective method for modeling systems composed of small sample sizes that contain a limited amount of information. This theory was proposed by Deng [25] in 1982 and has been widely used in the energy field. Since the time response function of the traditional grey prediction model $(\operatorname{GM}(1,1))$ is an approximately exponential function, the $\operatorname{GM}(1,1)$ model can obtain satisfactory prediction results when the data follows the law of quasi-exponent. However, the development trend of many things such as wind energy, solar energy, and geothermal energy does not strictly follow the law of quasi grey exponent but is accompanied by periodic disturbance (seasonal disturbance) [31] and irregular disturbance. Thus, the direct application of the $\operatorname{GM}(1,1)$ model to predict wind power generation cannot achieve the desired prediction results. Therefore, how to deal with the seasonal and long memory characteristics of the seasonal wind power capacity and improve the forecasting performance of the grey forecasting model becomes a key issue. To solve 
this problem, a new seasonal discrete grey prediction model is proposed in this paper. On the one hand, based on the moving average filtering algorithm, the proposed model realized the recognition of seasonal and trend features. On the other hand, based on the optimization of fractional order and initial value, the collaborative optimization of trend and season is realized. Therefore, compared with the traditional seasonal grey forecasting model, this model not only captures seasonal characteristics of the seasonal wind power generation but also can be applied to the data series of different periods.

\subsection{Literature review}

\subsubsection{Research progress on forecasting wind power generation}

The prediction of renewable energy has always been the focus of scholars' research. In the past, according to the different forecasting principles, the methods of wind power forecasting mainly included physical models [45], statistical models, and machine learning. For example, Based on the hub-height measurements collected from a fleet of turbines, Aziz Ezzat [11] made predictions about the short-term wind speed and power of a turbine-specific. Jacondino and Nascimento et al. studied the impact of different physical parameterizations in forecasting wind speeds at two onshore wind farms using the Weather Research and Forecasting (WRF) model [20]. Given the problem that Numerical Weather Prediction models are not applicable to heterogeneous terrain, by combining WRF mesoscale model and HDWind microscale model, Prieto-Herráez et al. [44] proposed a dynamic downscaling method to improve the local accuracy of wind speed prediction. However, the physical method is not suitable for short-term prediction because it requires a long time to run [64].

The statistical model mainly uses regression analysis and time series analysis methods. Commonly used models are ARMA, ARIMA, ARMAX, and ARX [10,26]. For example, considering the changes in Spatiotemporal wind power dynamics that are related to factors such as seasons or wind farm setup changes, Messner and Pinson proposed a time-adaptive lasso estimator and an effective coordinate descent algorithm to update the parameters of vector autoregression (VAR) model recursively and effectively track and simulate the dynamic changes of multivariate time series [39]. Compared with physical models, statistical models do not require extensive information to build models and are suitable for short-term forecasting. However, these models are difficult to deal with the nonlinear and uncertain characteristics of wind power.

Since Machine learning $[4,7]$ and data mining [28] can better capture the nonlinear characteristics of wind power, these models have become the common methods at the moment. Abhinav et al. propose a small wavelet neural network prediction model, which has strong robustness. The model can predict the short-term changes in wind power and has high prediction accuracy [2]. Machine learning and data mining, while showing advantages over traditional statistical techniques, also have their drawbacks, such as the ANN model sometimes being caught up in the dilemma of local minimum and overfitting, and are sensitive to parameter selection [18]. The selection of error penalty factors and kernel parameters significantly affects the prediction accuracy of the support vector machine model [40]. Considering the problems of machine learning models, scholars have also improved the methods from different perspectives. For example, given the problem that the hyperparameters and architecture optimization of deep neural networks (DNNs) leaned on experience, Jalali et al. adopted the enhanced version of the grasshopper optimization algorithm to optimize the architecture of deep long short-term memory (LSTM) neural network, so as to predict the wind speed [21]. And an extended fuzzy wavelet neural network (FWNN) is also extended for wind power forecasting [13].

Considering the advantages of these models, how to make full use of the advantages of each model to achieve the ideal prediction of wind power has become a hot research topic. For example, Lin et al. designed input features based on the physical process of offshore wind turbines, and further studied their linear and nonlinear correlations with wind power through deep learning algorithms [35]. Higashiyama et al. [16] used a three-dimensional convolutional neural network to extract spatiotemporal features of NWP data. Wang et al. realized the combination of Back Propagation Neural Network (BPNN), Radial Basis Function Neural Network (RBFNN), and Support Vector Machine (SVM) through integrated learning, which effectively improved the reliability of the model. In addition, the use of the Bayesian Model Average (BMA) also effectively reduces the 
uncertainty of a single model and prevents the overfitting of the trained model [54]. Kisvari et al. proposed a novel data-driven method based on the integration of data pre-processing \& re-sampling, anomalies detection $\&$ treatment, feature engineering, and hyperparameter tuning [27]. Besides, the complex quarterly fluctuation problem can be decomposed into sub-problems, and different models are used to predict each unobservable part separately. Because they exhibit more predictable dynamic behavior, some scholars use seasonal decomposition techniques to deal with the seasonal effects of data [5]. For example, Wang et al. decomposed the wind power sequence into trend sequence, harmonic sequence, and noise sequence through singular spectrum analysis, and combined the decomposed sequence with a new Laguerre neural network to predict, and obtained a relatively ideal result [55]. Nail et al. [42] combines the variational mode decomposition (VMD) and low-rank multi-kernel ridge regression (MKRR) to effectively predict the change interval of wind energy and wind speed. And the mixed model has better prediction performance than low-rank multi-kernel ridge regression based on empirical mode decomposition (EMD). Dhiman et al. proposed a hybrid machine intelligent wind forecasting model based on wavelet transform and Support Vector Regression [8]. And Abedinia et al. realized the prediction of wind power by inputting the signal decomposed by improved empirical mode decomposition (IEMD) into a hybrid model based on bagging neural network combined with K-means clustering [1]. Yildiz et al. developed a novel residual-based convolutional neural network model for wind power forecasting based on VMD [63].

As we have seen, these methods have excellent performance in predicting short-term wind power. However, several limitations of machine learning approaches concern the large number of observations that are required to calibrate model parameters and the challenge of an internal structure that is unknown [60]. Moreover, most of the above works focus on the short-term forecasting of wind power, while the mid-to-long term prediction of wind power is also of great significance to the grid balance, the adjustment of energy structure, and the formulation of energy policy. In China, the government only counts the monthly wind power generation from September 2009 to now, and the solar power generation only counts the monthly data from May 2016 to the present. Thus, concerning the mid-to-long term prediction of wind power in China, the available data seem to be insufficient for some machine learning approaches whereas the grey forecasting theory can effectively address problems with limited samples.

\subsubsection{Application of grey model in energy prediction}

The grey system theory is a method for solving the modeling of small sample data proposed by Deng [25] in 1982. It makes full use of existing information by mining and generating limited information. The GM(1,1) model is a classic model for predicting small sample data with insufficient information. When we are unable to obtain sufficient data or only understand part of the internal information of the system, the grey prediction model can often achieve the desired effect. Therefore, at present, the grey prediction model has been widely used in the field of energy prediction [43,59]. For example, Liu et al. predicted the primary energy consumption of Spain by establishing a grey neural network and input-output combined forecasting model, which realized the series combination of the grey prediction model and neural network model by taking the prediction results of the grey prediction model as the input of the neural network model [37]. Zhou et al. proposed a new discrete grey model, taking into account the nonlinear and volatility, and predicted the natural gas consumption in Jiangsu Province, China [67]. Jia et al. used the Markov chain forecasting method to revise the forecast value of the $\operatorname{GM}(1,1)$ model to predict coal consumption in Gansu, China [23]. Guefano et al. proposed a new hybrid GM(1,1)-VAR(p) model based on the Grey and Vector autoregressive methods [14]. And Şahin proposed a novel optimized fractional nonlinear grey Bernoulli model by optimizing background value, power index value, and fractional order value with the genetic algorithm method [48]. Javed et al. improved the existing grey model by optimizing fractional-order value and background value [22]. Moreover, Moonchai et al. developed a modified grey model with a Kalman filter and applied the model to renewable energy consumption [41].

Since the traditional GM(1,1) model is suitable for predicting stable sequences of single growth trends, the accuracy of the prediction of time series with quarterly fluctuations is significantly decreasing. To eliminate seasonal fluctuations, scholars have adopted several methods to make improvements. For example, Xiao et al. proposed a seasonal grey model based on periodic truncate cumulative generation operations [61]. Qian and Dang 
transformed the non-monotonic oscillation sequence into a monotonic sequence by accelerating the translation transformation of the original data, thereby improving the prediction accuracy [46]. By using HP filter to separate the trend component and seasonal component of the original time series, Qian and Wang used GM( 1,1$)$ model to predict the trend component, and then calculated the seasonal index to adjust the predicted value of the trend, thus realizing the prediction of China's wind power generation [47]. Based on the GM(1,1) model, Wang et al. introduced the concept of seasonal fluctuations and established the $\operatorname{SFGM}(1,1)$ model [49]. Considering that the traditional $\operatorname{GM}(1,1)$ model is only suitable for time series with small fluctuations, Wang et al. established a DGGM(1,1) model based on data grouping to predict quarterly hydropower production in China [50]. And Wang et al. proposed a grey predictive model based on data grouping operators. The models are grouped by month (quarter) and buffered separately to handle the prediction errors caused by seasonal fluctuations [52]. To accurately forecast the seasonal change of electricity consumption in the major economic sector, Wang et al. proposed a seasonal grey model based on the accumulation operations generated by seasonal factors [51]. Then, Wang et al. considered that the seasonal adjustment factors of the model are static, so he proposed a seasonal grey model based on dynamic seasonal adjustment factors [53]. In addition, the methods of integrating annual trends and monthly seasonal characteristics of data series [32] and improving the adaptation to seasonal time series from the perspective of model structure [9] are the latest new methods. In general, these methods focus on the seasonal adjustment of the original data and the optimization of the grey forecast model. The former enhances the stability of data, while the latter flexibly reflect the characteristics of seasonal fluctuations.

Grey forecasting technology has a good application prospect in the field of energy forecasting, and it provides a valuable decision basis for energy planning. Also, the development and extension of the grey prediction model are conducive to the prediction of other things with similar characteristics. Nevertheless, the grey prediction model for seasonal time series still has some shortcomings. Firstly, most seasonal grey prediction models use the seasonal index to adjust the seasonality of time series, but the calculation method of the seasonal index is not suitable for time series with the long-term trend. Secondly, the seasonal grey prediction model tends to adopt the traditional GM(1,1) model as the basic model of the new seasonal model, but the problem of background value, initial value, and jump between parameter estimation and prediction formula of traditional $\operatorname{GM}(1,1)$ model is ignored. Thirdly, it is difficult to describe the long memory character of time series and to satisfy the criterion of new information priority only by using traditional GM(1,1). Finally, although most of the seasonal grey prediction models have been improved in capturing the seasonal and trend characteristics, the optimization of the seasonal grey prediction model, such as the selection of fractional order and the optimization of $\operatorname{GM}(1,1)$ model, lacks the comprehensive consideration of the seasonal and trend characteristics of the time series.

To solve these problems, based on the seasonal and long memory characteristics of the seasonal wind power generation series, this paper adopts the moving average filtering algorithm and the Particle swarm optimization to establish the seasonal grey prediction model based on collaborative optimization (COSGM). On the one hand, the model is based on moving average filtering algorithm to realize the recognition of seasonal and trend features. On the other hand, based on the optimization of fractional order and initial value, the collaborative optimization of trend and season is realized. In addition, the introduction of the discrete grey prediction model also solves the problem of the traditional grey prediction model hopping between the discrete forms with continuous forms and improves the applicability and scope of the model.

The rest of the paper is arranged as follows: Section 2 introduces the proposed model. Section 3 makes an empirical analysis based on China's seasonal wind power generation data from 2012Q1 to 2020Q1, and further uses the proposed model to forecast the seasonal wind power generation from 2021 to 2024. Conclusions and discussions are drawn in Section 4.

\section{Methods}

In this section, we introduce the underlying model and the overall process of modeling for the COSGM model. Besides, the relevant background knowledge is described in detail. And the COSGM model proposed in this paper is improved based on $\operatorname{DGM}(1,1)$ model, so one of the main contributions of the proposed model lies 


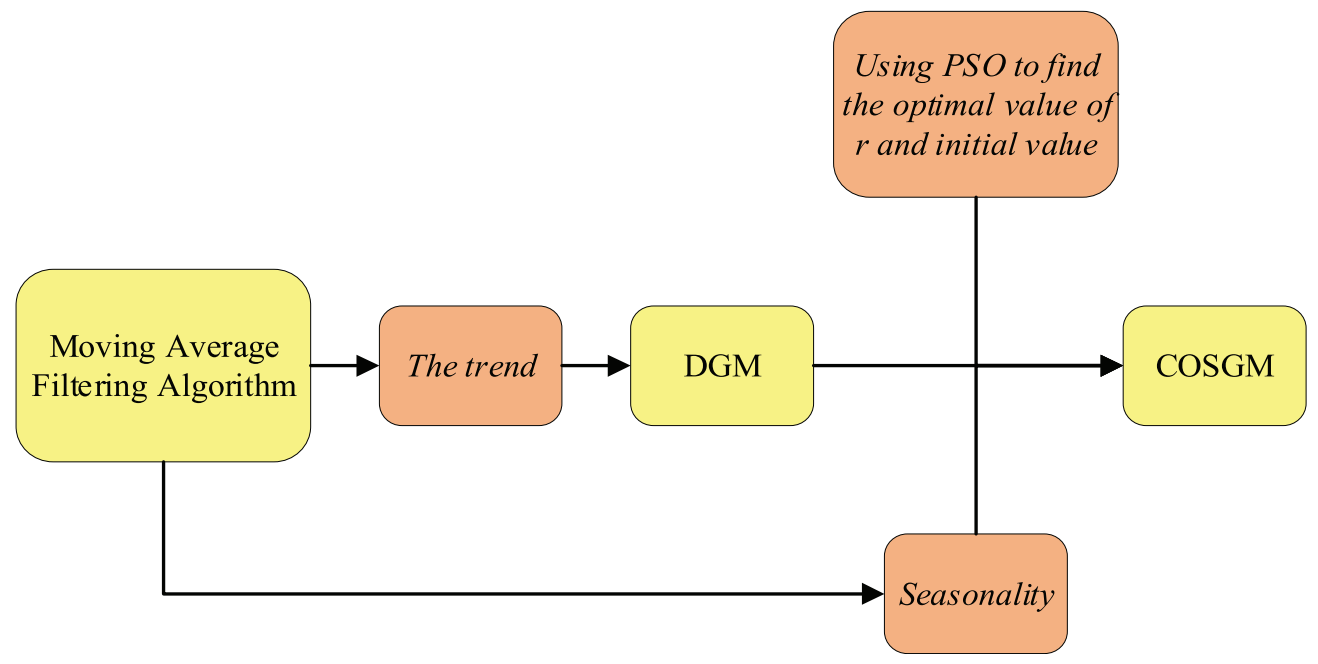

FIGURE 2. Correlation between models.

in effectively avoiding the structural weakness in transforming discrete data into continuous series to mitigate the information distortion. In addition, the particle swarm optimization (PSO) algorithm is further applied to optimize the introduced fractional-order and initial value simultaneously, which significantly improves the ability to capture the trend features of time series. Moreover, the introduction of the moving average filtering algorithm also makes the trend and seasonality clear, which lays a foundation for improving the seasonal adaptability of the grey prediction model. The improvement process and the correlation among models are shown in Figure 2. The modeling process of the proposed model is described in detail below.

\subsection{The traditional $\operatorname{GM}(1,1)$ model}

Grey system theory is an important method for modeling small sample sizes with insufficient information. Through the mining of grey information, the theory can make full use of existing information. The GM $(1,1)$ model is the most classic in grey forecasting theory. The model weakens the randomness of the data sequence by using the accumulative generating operator, thereby mining the long-term trend of the data sequence. The modeling process of the model is as follows.

First, suppose $X^{(0)}=\left(x^{(0)}(1), x^{(0)}(2), x^{(0)}(3), \cdots, x^{(0)}(n)\right)$ is the original data series affected by the season.

Then, by applying the first-order accumulation generation (1-AGO) to the original data sequence, we can get the first-order cumulative generating sequence $X^{(1)}=\left(x^{(1)}(1), x^{(1)}(2), x^{(1)}(3), \cdots, x^{(1)}(n)\right)$ where,

$$
x^{(1)}(k)=\sum_{i=1}^{k} x^{(0)}(i), \quad k=1,2, \cdots, n .
$$

Then, the mean series can be calculated:

$$
z^{(1)}(t)=0.5 x^{(1)}(t)+0.5 x^{(1)}(t-1), \quad t=2,3, \cdots, n .
$$

Based on the above sequence, we can establish a first-order univariate differential equation as a prediction model, namely the GM(1,1) model. The standard form of the grey difference equation is:

$$
x^{(0)}(t)+z^{(1)}(t)=b, \quad t=2,3, \cdots, n .
$$


Then the corresponding whitening differential equation is:

$$
\frac{\mathrm{d} x^{(1)}(t)}{\mathrm{d} t}+a x^{(1)}(t)=b
$$

The parameters $a$ and $b$ can be estimated by the following formula

$$
P=[a, b]^{T}=\left(B^{T} B\right)^{-1} B^{T} Y
$$

where, $B=\left[\begin{array}{ll}-z^{(1)}(2) & 1 \\ -z^{(1)}(3) & 1 \\ -z^{(1)}(4) & 1 \\ \vdots & \vdots \\ -z^{(2.1)}(n) & 1\end{array}\right], Y=\left[\begin{array}{l}x^{(0)}(2) \\ x^{(0)}(3) \\ x^{(0)}(4) \\ \vdots \\ x^{(0)}(n)\end{array}\right]$.

The time-response function of the $\operatorname{GM}(1,1)$ model is:

$$
\hat{x}^{(1)}(k)=\left(x^{(0)}(1)-\frac{b}{a}\right) e^{-a(k-1)}+\frac{b}{a}, \quad k-2,3, \cdots, n .
$$

Finally, we can get the predicted value of the original sequence by the first-order inverse accumulation generation operation (IAGO) (Eq. (2.7)). It can be seen from equation $(2.7)$ that the traditional GM(1,1) model has higher prediction accuracy for monotonically increasing or decreasing exponential sequences. If there are constant disturbances and periodic disturbances in the data sequence, the traditional GM(1,1) model is difficult to achieve the ideal prediction effect.

$$
\begin{aligned}
\hat{x}^{(0)}(k+1) & =\hat{x}^{(1)}(k+1)-\hat{x}^{(1)}(k) \\
& =\left(x^{(0)}(1)-\frac{b}{a}\right)\left(1-e^{a}\right) e^{-a k}, \quad k=2,3, \cdots, n .
\end{aligned}
$$

\subsection{The basic DGM $(1,1)$ model}

The traditional $\operatorname{GM}(1,1)$ model uses a discrete equation to estimate the parameters, while the prediction equation obtained from the continuous equation is used in forecasting. There are structural errors in forecasting. Thus, Xie and Liu proposed a discrete grey forecasting model [62].

First, suppose $X^{(0)}=\left(x^{(0)}(1), x^{(0)}(2), x^{(0)}(3), \cdots, x^{(0)}(n)\right)$ is the original data series affected by the season.

Then, by applying the first-order accumulation generation (1-AGO) to the original data sequence, we can get the first-order cumulative generating sequence $X^{(1)}=\left(x^{(1)}(1), x^{(1)}(2), x^{(1)}(3), \cdots, x^{(1)}(n)\right)$ based on equation (2.1).

Based on the $X^{(1)}$, the $\operatorname{DGM}(1,1)$ model is presented as:

$$
x^{(1)}(k+1)=\beta_{1} x^{(1)}(k)+\beta_{2} .
$$

The parameters $\beta_{1}$ and $\beta_{2}$ can be estimated by the following formula

$$
P=\left[\beta_{1}, \beta_{2}\right]^{T}=\left(B^{T} B\right)^{-1} B^{T} Y
$$

where, $B=\left[\begin{array}{ll}x^{(1)}(2) & 1 \\ x^{(1)}(3) & 1 \\ x^{(1)}(4) & 1 \\ \vdots & \vdots \\ x^{(1)}(n-1) & 1\end{array}\right], Y=\left[\begin{array}{c}x^{(1)}(2) \\ x^{(1)}(3) \\ x^{(1)}(4) \\ \vdots \\ x^{(1)}(n)\end{array}\right]$. 
Then, by interating equation (2.8), the time response function can be reckoned as:

$$
\hat{x}^{(1)}(k+1)=\beta_{1}^{k} x^{(0)}(1)+\frac{1+\beta_{1}^{k}}{1-\beta_{1}} \beta_{2} .
$$

Finally, we can get the predicted value of the original sequence by the first-order inverse accumulation generation operation (IAGO) (Eq. (2.11)).

$$
\begin{aligned}
\hat{x}^{(0)}(k+1) & =\hat{x}^{(1)}(k+1)-\hat{x}^{(1)}(k) \\
& =\beta_{1}^{k}\left(1-\beta_{1}^{-1}\right)\left(x^{(0)}(1)-\frac{\beta_{2}}{1-\beta_{1}}\right) .
\end{aligned}
$$

Further, the growth rate of the estimated value can be calculated:

$$
\hat{v}(k)=\frac{\hat{x}^{(0)}(k)-\hat{x}^{(0)}(k-1)}{\hat{x}^{(0)}(k-1)}=\hat{\beta}_{1}-1 .
$$

Equation (2.12) implies the forecasted results yielded by the $\operatorname{DGM}(1,1)$ model vary at a fixed rate, which leads to high accuracy when applying the $\operatorname{DGM}(1,1)$ model to simulate the quasi-homogeneous exponential sequences. If there are constant disturbances and periodic disturbances in the sequence, the DGM(1,1) model is difficult to achieve the ideal prediction effect.

\subsection{The seasonal $\operatorname{GM}(1,1)$ model}

To enhance the forecasting ability of the grey forecast model for seasonal time series, Wang et al. introduced seasonal factors into the accumulating generation process. By changing the internal mechanism of the grey model, Wang established a grey prediction model based on the seasonal accumulation operator [51]. The modeling process is as follows.

First, suppose $X^{(0)}=\left(x^{(0)}(1), x^{(0)}(2), x^{(0)}(3), \cdots, x^{(0)}(n)\right)$ is the original data series affected by the season.

By the sequence operator $S$, we can get the sequence $X_{s}^{(1)}=X^{(0)} S=\left(x^{(1)}(1) s, x^{(1)}(2) s, \cdots, x^{(1)}(n) s\right)$, where

$$
x^{(1)}(k) s=\sum_{i=1}^{k} x^{(0)}(i) / f_{s}(i), \quad k=1,2, \cdots, n .
$$

$S$ is the first-order seasonal accumulating generation operator (1-SAGO). In equation $(2.13), f_{s}(i)$ is the seasonal adjustment factor at the $i$ th time point, reflecting the degree to which the actual value deviates from the average value of the trend due to seasonal influence. $f_{s}(i)$ can be calculated by equation (2.14).

$$
f_{s}(i)=\frac{\bar{x}_{M}^{(0)}(i)}{\bar{x}_{M N}^{(0)}(i)} .
$$

In equation (2.14), $M$ and $N$ respectively represent the number of seasonal cycles in a year and the year at the $i$ th time point. $\bar{x}_{M}^{(0)}(i)$ is the quarterly average of the $i$ th time point, and $\bar{x}_{M N}^{(0)}(i)$ is the total average of all quarters.

Based on the above, the background value sequence can be calculated as follows:

$$
z_{s}^{(1)}(k)=0.5 x_{s}^{(1)}(k)+0.5 x_{s}^{(1)}(k-1), \quad k=2,3, \cdots, n
$$

the grey differential equation can be constructed as follows:

$$
x^{(0)}(k) / f_{s}(k)+a_{s} z_{s}^{(1)}(k)=b_{s}, \quad k=2,3, \cdots, n .
$$


On this basis, we can get the whitening differential equation:

$$
\frac{\mathrm{d} x_{s}^{(1)}(t)}{\mathrm{d} t}+a_{s} x_{s}^{(1)}(t)=b_{s} .
$$

Then

$$
\hat{a}_{s}=\left(a_{s}, b_{s}\right)^{T}=\left(B_{s}^{T} B_{s}\right)^{-1} B_{s}^{T} Y_{s}
$$

we can get the values of parameters $a_{s}$ and $b_{s}$.

And the prediction formula of the $\operatorname{SGM}(1,1)$ model is

$$
\hat{x}^{(1)}(k)=\left[x^{(0)}(1) / f_{s}(1)-\frac{b_{s}}{a_{s}}\right] e^{-a_{s}(k-1)}+\frac{b_{s}}{a_{s}}, \quad k=2,3, \cdots, n .
$$

Finally, we can get the predicted value of the original sequence by the first-order inverse accumulation generation operation (IAGO) (Eq. (2.22)).

$$
\hat{x}^{(0)}(k+1)=f_{s}(k+1)\left(\hat{x}_{s}^{(1)}(k+1)-\hat{x}_{s}^{(1)}(k)\right), \quad k=2,3, \cdots, n .
$$

From equation (2.14) we can see that when the time series data does not have obvious long-term trends and cyclic fluctuations, the prediction accuracy of the $\operatorname{SGM}(1,1)$ model is better [36].

\subsection{Moving average filtering algorithm}

Generally, a complex time series is composed of many mutually influencing components, so it is difficult to directly establish a forecast model for complex time series. At present, scholars widely use the method of decomposing time series to separately establish forecasting models for time series [3,15]. For example, Qian and Wang used GM(1,1) model to predict the trend component and then calculated the seasonal index to adjust the predicted value of the trend, thus realized the prediction of China's wind power generation [47]. Generally, it is assumed that the time series is composed of four parts: trend component, cyclic component, periodic component, and an irregular component. And the HP filter assumes that the time series consists of two parts: the trend component and the cyclic component [17].

To further reveal the long-term trend and seasonal characteristics of wind power generation, this study establishes a seasonal grey forecast model based on a moving average filter.

The principle of the moving average filter is that the original data sequence $X_{t}$ is transformed by a function, that is, filtered by the moving average filter, some unwanted information can be eliminated and a new data sequence $Y_{t}$ can be obtained. The essence of the moving average filter is to generate the value of the filtered data sequence at time $t$ by using the weighted average results of $N$ samples data from $p$ samples before time $t$, $f$ samples after time $t$, and sample at time $t$. That is

$$
M\left(X_{t}\right)=\sum_{k=-p}^{+f} \theta_{k} X_{t+k}
$$

where $N=p+f+1$. And when $f=p$, the filter is called an asymmetric moving average filter.

Overall, a single symmetric equal-weight moving average filter with a specific number of terms can smooth out the periodic fluctuations of the corresponding frequency (the number of terms must be greater than or equal to the period length of the fluctuation). For seasonal fluctuations of quarterly (monthly) data with a period of 4 (12), we can eliminate seasonality well by using symmetrical equal-weight moving average filtering with an item number of 4 (12). However, when $N=4$ (12), the number of items is even, and an asymmetric equal-weight moving average filter cannot be constructed. Therefore, according to the principle of the multi-pass filter, the X11 seasonal adjustment theory constructs a $2 \times N$ term symmetric moving average filter. For the 
quarterly data with a period of 4 (12), the X11 seasonal adjustment theory constructs a 5 (13) term symmetric non-equal-weight filter.

The corresponding weight coefficient is

$$
\theta_{k}=\{1,2,2,2,1\} / 8 \quad\left(\theta_{k}=\{1,2,2,2,2,2,2,2,2,2,2,2,1\} / 12\right) .
$$

Since the filter can well satisfy three conditions: eliminating seasonality, retaining long-term trend, and smoothing irregular fluctuation [65], we can make an ideal description of the long-term trend and seasonal characteristics of the original time series.

In general, seasonal factors acting on time series are relatively stable, so we can calculate seasonal adjustment factors by using the average degree to which the actual value deviates from the long-term trend value due to seasonal factors. That is

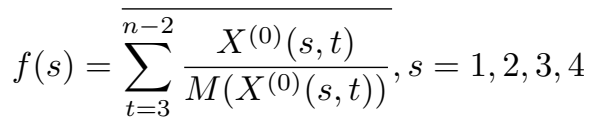

where $s$ refers to the number of cycles, $X^{(0)}(s, t)$ and $M\left(X^{(0)}(s, t)\right)$ represent the actual data obtained from the original time series and the data filtered by the moving average filter (the long-term trend) respectively. And $X^{(0)}(s, t)$ represents the actual value for time point $t$, quarter $s$. Moreover, $f(s)$ indicates the seasonal factor acting time point $t$, quarter $s$.

\subsection{COSGM model}

Considering that the traditional grey forecasting model is not good at capturing the seasonal and trend characteristics, this paper constructs the COSGM model. Then, in this paper, based on the recognition of seasonal time series seasonality and trend characteristics by using the moving average filter algorithm, a fractional-order discrete gray prediction model with initial value correction is first constructed for trend characteristics, and then a COSGM model is established by using seasonal factors to correct forecast trend. PSO algorithm is mainly used to modify the initial value and determine the fractional order. Thus, the proposed model takes into account the long memory and seasonal characteristics of the time series, satisfies the principle of new information priority, and improves the initial value dependence of the grey prediction model. The definitions involved in the COSGM model are shown below. And the schematic flowchart of the COSGM model is depicted in Figure 3.

\subsubsection{The establishment of the COSGM model}

Definition 2.1. It is assumed that $X^{(0)}=\left\{x^{(0)}(s, 1), x^{(0)}(s, 2), \cdots, x^{(0)}(s, n)\right\}$ is the original data series, for which the data points are non-negative and equally spaced over time. $x^{(0)}(s, n)$ represents the actual value for time point $n$, quarter $s$. Then the data sequence $X_{T}^{(0)}=\left\{x_{T}^{(0)}(s, 1), x_{T}^{(0)}(s, 2), \cdots, x_{T}^{(0)}(s, n)\right\}$ obtained by moving average filtering is called trend sequence, and the corresponding seasonal factor is $f(s)$, where $s$ refers to the number of cycles. $X_{T}^{(0)}$ and $f(s)$ represents the long-term development trend of the system and the average degree to which the actual value deviates from the long-term trend value due to seasonal factors, respectively. Moreover, $f(s)$ indicates the seasonal factor acting time point $n$, quarter $s$.

Definition $2.2([58])$. Given $X_{T}^{(0)}=\left\{x_{T}^{(0)}(s, 1), x_{T}^{(0)}(s, 2), \cdots, x_{T}^{(0)}(s, n)\right\}$, then the $r$-order accumulated generating sequence $\left(r\right.$-AGO) is $X_{T}^{(r)}=\left\{x_{T}^{(r)}(s, 1), x_{T}^{(r)}(s, 2), \cdots, x_{T}^{(r)}(s, n)\right\}$.

Its calculation formula is

$$
x_{T}^{(r)}(s, k)=\sum_{j=1}^{k}\left(\begin{array}{c}
k-j+r-1 \\
k-j
\end{array}\right) x_{T}^{(0)}(s, j), \quad k=1,2, \cdots, n
$$




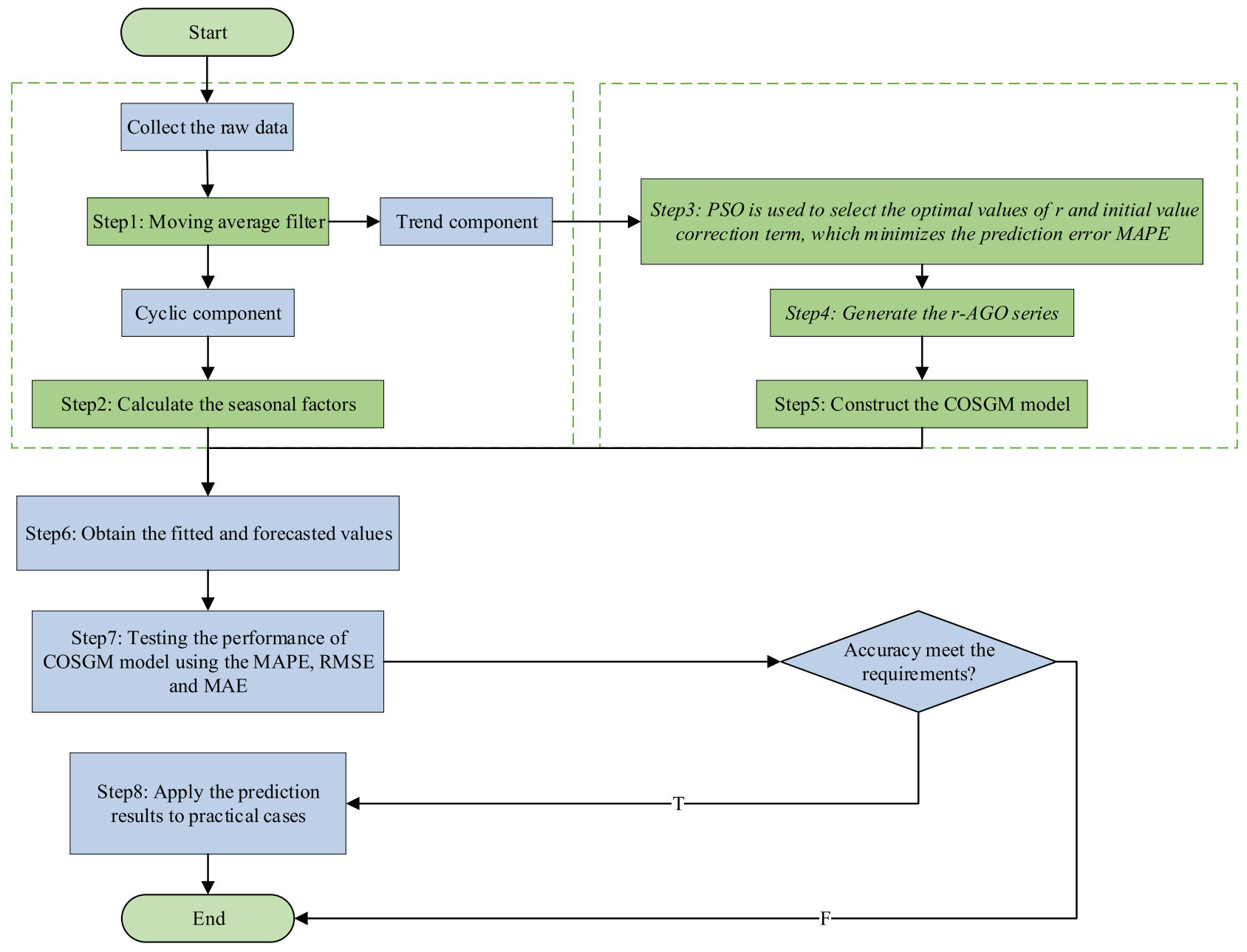

Figure 3. Schematic diagram of the COSGM model.

where $\left(\begin{array}{c}r-1 \\ 0\end{array}\right)=1,\left(\begin{array}{c}k-1 \\ k\end{array}\right)=0,\left(\begin{array}{c}k-j+r-1 \\ k-j\end{array}\right)=\frac{(k-j+r-1)(k-j+r-2) \cdots(r+1) r}{(k-j) !}$ is the general Newton binomial coefficient. In equation (2.24), $r$ indicates the fractional-order value. And fractional derivatives accumulate the whole history of the system in weighted form. Moreover, when $r=1, x_{T}^{(r)}(s, k)$ reduces to $x_{T}^{(r)}(s, k)=\sum_{j=1}^{k} x_{T}^{(0)}(s, j)$ which denotes the first-order accumulated generating operation sequence of $X_{T}^{(0)}$.

Definition $2.3([38])$. Given $X_{T}^{(0)}=\left\{x_{T}^{(0)}(s, 1), x_{T}^{(0)}(s, 2), \cdots, x_{T}^{(0)}(s, n)\right\}$, then the $r$-order inverse accumulated generating sequence $\left(r\right.$-IAGO) is $X_{T}^{(-r)}=\left\{x_{T}^{(-r)}(s, 1), x_{T}^{(-r)}(s, 2), \cdots, x_{T}^{(-r)}(s, n)\right\}$.

Its calculation formula is

$$
x_{T}^{(-r)}(s, k)=\sum_{j=1}^{k}\left(\begin{array}{c}
k-j-r-1 \\
k-j
\end{array}\right) x_{T}^{(0)}(s, j), \quad k=1,2, \cdots, n
$$

when $r=-1$, the $r$-IAGO just yields the first-order difference. 
Definition 2.4 ([33]). Based on the $r$-AGO series, the $\operatorname{DGM}^{r}(1,1)$ model is presented as:

$$
x_{T}^{(r)}(s, k+1)=\beta_{1} x_{T}^{(r)}(s, k)+\beta_{2} .
$$

Consequently, the parameters $\hat{\beta}=\left[\hat{\beta}_{1}, \hat{\beta}_{2}\right]^{T}$ of the $\operatorname{DGM}^{r}(1,1)$ model can be estimated by utilizing the least square method (LSM). That is

$$
\hat{\beta}=\left[\hat{\beta}_{1}, \hat{\beta}_{2}\right]^{T}=\left(B^{T} B\right)^{-1} B^{T} Y
$$

where $B=\left[\begin{array}{cc}x_{T}^{(r)}(s, 1) & 1 \\ x_{T}^{(r)}(s, 2) & 1 \\ \vdots & \vdots \\ x_{T}^{(r)}(s, n) & 1\end{array}\right]$ and $Y=\left[\begin{array}{c}x_{T}^{(r)}(s, 2) \\ x_{T}^{(r)}(s, 3) \\ \vdots \\ x_{T}^{(r)}(s, n+1)\end{array}\right]$.

Definition 2.5. Based on the parameters $\hat{\beta}=\left[\hat{\beta}_{1}, \hat{\beta}_{2}\right]^{T}$, the time response function of the $\operatorname{DGM}^{r}(1,1)$ model can be calculated by

$$
\hat{x}_{T}^{(r)}(s, k+1)=\hat{\beta}_{1}^{k} x_{T}^{(r)}(s, 1)+\frac{1-\hat{\beta}_{1}^{k}}{1-\hat{\beta}_{1}} \hat{\beta}_{2}, \quad k=1,2, \cdots, n .
$$

In equation $(2.28), x_{T}^{(r)}(s, 1)$ is called the iterative datum. And the $\operatorname{DGM}^{r}(1,1)$ model in Definition 2.3 is based on the hypothesis that the first datum of the sequence is invariable. Actually, the hypothesis violates with the fact [62]. Thus, considering that the iterative datum value significantly influences the simulative and predictive results [34], we set the iterative datum value as $x_{T}^{(r)}(s, 1)+\varsigma$. And we solve this sensitive problem of iterative datum value by the optimum method. Therefore, the new time response function can be reckoned as:

$$
\hat{x}_{T}^{(r)}(s, k+1)=\hat{\beta}_{1}^{k}\left(x_{T}^{(r)}(s, 1)+\varsigma\right)+\frac{1-\hat{\beta}_{1}^{k}}{1-\hat{\beta}_{1}} \hat{\beta}_{2}, \quad k=1,2, \cdots, n
$$

where $\varsigma$ is the initial value correction term.

Definition 2.6 ([38]). Via the $r$-order inverse accumulation generation operator ( $r$-IAGO), the predicted values of $\operatorname{DGM}^{r}(1,1)$ model can be deduced:

$$
\hat{X}_{T}^{(0)}=\sum_{i=1}^{k}\left(\begin{array}{c}
k-i-r-1 \\
k-i
\end{array}\right) \hat{x}_{T}^{(r)}(s, i), \quad k=1,2, \cdots, n .
$$

In equation $(2.30), \hat{X}_{T}^{(0)}$ is the restored value of the development trend of the system.

Definition 2.7. Given $\hat{X}_{T}^{(0)}$ and $f(s)$, then the COSGM model is defined as:

$$
\hat{X}^{(0)}=f(s) \cdot \hat{X}_{T}^{(0)}=\left\{f(s) \cdot \hat{x}_{T}^{(0)}(s, 1), f(s) \cdot \hat{x}_{T}^{(0)}(s, 2), \cdots, f(s) \cdot \hat{x}_{T}^{(0)}(s, n), \cdots\right\} .
$$

Moreover, the restored values of $X^{(0)}$ is $\hat{X}^{(0)}$. When $f(s)=1$, the COSGM model reduces to the traditional $\operatorname{DGM}^{r}(1,1)$ model. 
TABLE 1. Measuring prediction accuracy with MAPE [30].

\begin{tabular}{llll}
\hline \hline MAPE (\%) & Forecasting ability & MAPE $(\%)$ & Forecasting ability \\
\hline$<10$ & High ability & $20-50$ & Reasonable ability \\
$10-20$ & Good ability & $>50$ & Weak ability \\
\hline
\end{tabular}

\subsubsection{Parameters estimation}

In the COSGM model, the order $r$ of fractional order and initial value correction term $\varsigma$ affect the prediction accuracy of the model significantly. The main idea of finding the optimal value of $r$ and $\varsigma$ is to minimize the error of the COSGM model. The mean absolute percentage error (MAPE) is usually used to represent the model error (the calculation of MAPE is given in Sect. 2.5.3). To find the optimal $r$ and $\varsigma$, this paper selects the PSO algorithm to optimize the parameters of this COSGM model. We can find the optimum values of the two coefficients by minimizing the mean absolute percentage error (MAPE) between the fitted and the real values. Thus, the objective function is constructed as follows.

$$
\begin{aligned}
& \min \text { MAPE }=\frac{1}{n} \sum_{k=1}^{n} \frac{\left|\hat{x}^{(0)}(k)-x^{(0)}(k)\right|}{x^{(0)}(k)} \\
& \text { s.t. }\left\{\begin{array}{l}
\hat{\beta}=\left[\hat{\beta}_{1}, \hat{\beta}_{2}\right]^{T}=\left(B^{T} B\right)^{-1} B^{T} Y \\
\hat{x}^{(0)}(k)=f(s) \cdot \hat{x}_{T}^{(0)}(s, k), \quad k=1,2, \cdots, n .
\end{array}\right.
\end{aligned}
$$

\subsubsection{Validation criterion for modeling efficacy}

To accurately evaluate the effectiveness of the COSGM model, three indexes were selected to evaluate the prediction accuracy of the model (the root mean square error (RMSE), mean absolute error (MAE) and mean absolute percentage error (MAPE)). These indicators are calculated as follows, respectively:

$$
\begin{aligned}
\text { RMSE } & =\sqrt{\frac{1}{n} \sum_{t=1}^{n} e^{(2)}(t)} \\
\text { MAE } & =\frac{1}{n} \sum_{t=1}^{n}|e(t)| \\
\text { MAPE } & =\frac{1}{n} \sum_{t=1}^{n}\left|\frac{e(t)}{x^{(0)}(t)}\right| \times 100 \%
\end{aligned}
$$

where $e(t)=x^{(0)}(t)-\hat{x}^{(0)}(t) . x^{(0)}(t)$ and $\hat{x}^{(0)}(t)$ represent actual and predicted values, respectively. Besides, the prediction accuracy can be graded using MAPE, as shown in Table 1. This is the criterion to determine the acceptability of the prediction error.

\section{EXPERIMENT: THE CASE STUDY}

With the rapid development of the economy, the energy demand is increasing day by day in China. However, the high consumption rate of non-renewable resources has a significant environmental impact on air quality and climate that cannot be ignored. To promote energy conservation and emissions reduction, Chinese officials have pledged to cut China's carbon intensity by $60-65 \%$ by 2030 compared with 2005 levels [66]. Therefore, renewable energy represented by wind and solar energy has been developed rapidly. The ideal forecast of Wind 


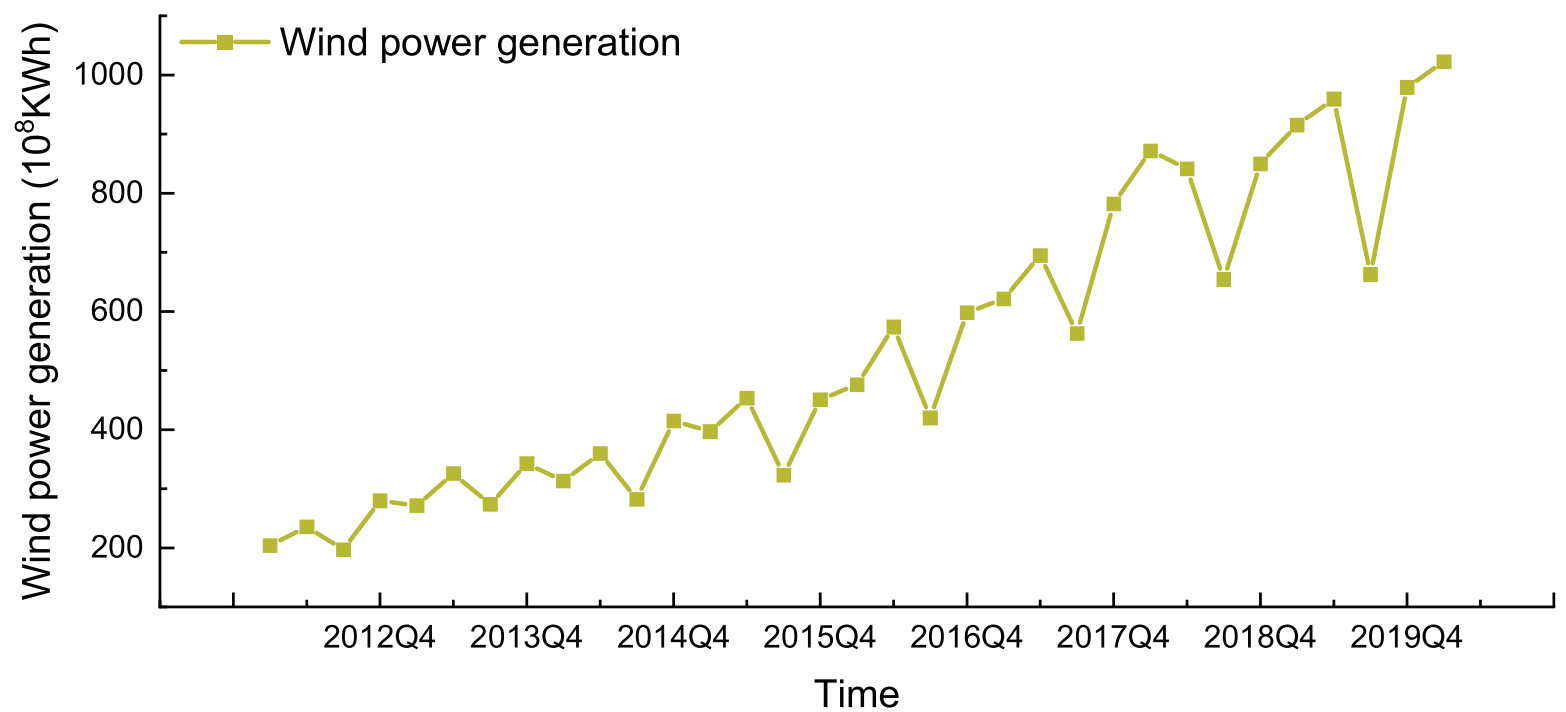

Figure 4. China's wind power generation from 2012Q1 to 2020Q1.

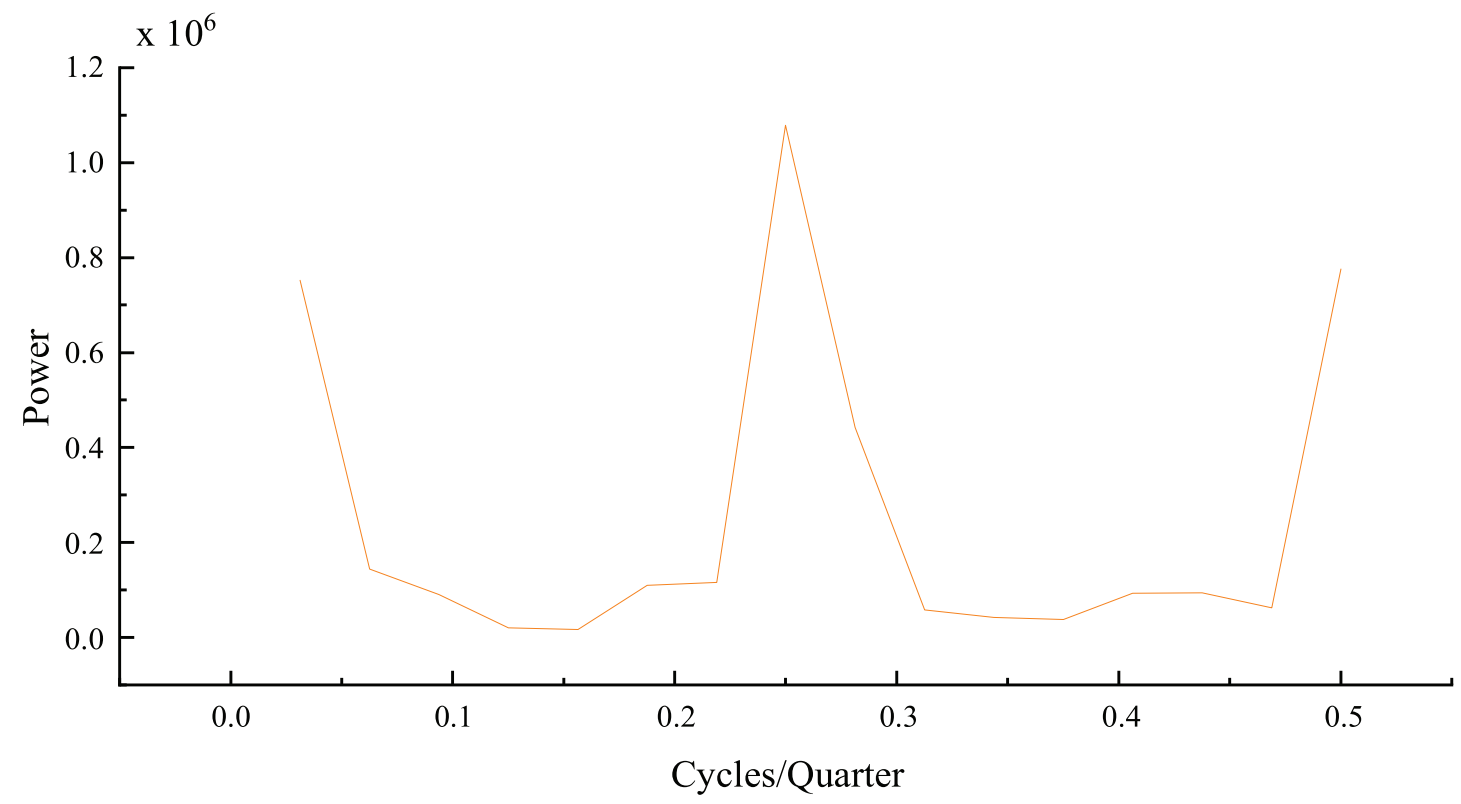

Figure 5. Power spectrum diagram based on FFT analysis.

power generation in China is helpful for the optimization of energy structure and the formulation of energy policy.

The power spectrum obtained by the Fast Fourier Transform (FFT) is shown in Figure 5. From Figures 4 and 5, China's wind power generation has been increasing from 2012 to 2020 and has a significant seasonal fluctuation, that is, $s=4$. Then, we can get the Hurst exponent $H=0.74$, that is, the wind power generation sequence shows long memory characteristics [56]. And the detailed data is gathered from China's 


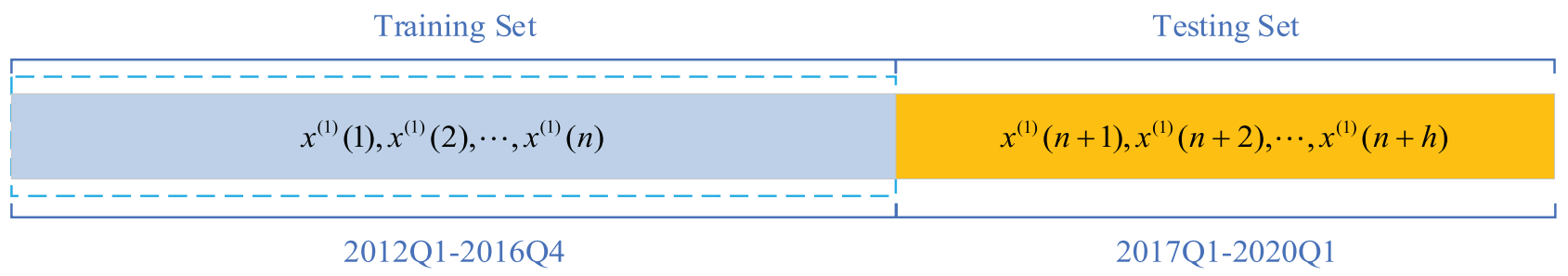

Figure 6. Data set partition diagram.

National Bureau of Statistics (https://data.stats.gov.cn/easyquery.htm?cn=A01). If we want to accurately predict future changes in China's wind power generation, it is necessary to consider these characteristics. Namely, seasonal and trend characteristics of wind power generation must be considered. Thus, the COSGM model is proposed to predict wind power generation in China. Firstly, to verify whether the prediction accuracy of this model can achieve the ideal effect, we conducted an empirical analysis of China's quarterly wind power generation from 2012Q1 to 2020Q1. The prediction results of the COSGM model were compared with those of the classical $\operatorname{GM}(1,1)$ model, Holt-Winters model, and $\operatorname{SGM}(1,1)$ model. In this study, the original data from 2012Q1 to 2020Q1 are divided into two sample sets. Data from 2012Q1 to 2016Q4 are divided into a training set, and data from 2017Q1 to 2020Q1 are divided into the test set. The data used in the study were all from the National Bureau of Statistics of China. Then, since the COSGM model is suitable for modeling wind power generation in China, the COSGM model is used to forecast wind power generation in China from 2021Q2 to 2024Q2.

\subsection{Model establishment}

To highlight the superiority of the COSGM modeling method, the dataset is divided into two groups. Data from 2012Q1 to 2016Q4 are divided into a training set, and data from 2017Q1 to 2020Q1 are divided into the test set (Fig. 6). And the prediction results of the COSGM model were compared with those of the classical $\operatorname{GM}(1,1)$ model, Holt-Winters model, and $\operatorname{SGM}(1,1)$ model. The prediction accuracy and practicability of the model for seasonal data series are verified.

\subsubsection{Direct modelling: traditional GM(1,1) model}

According to the modeling procedure used in the traditional $\operatorname{GM}(1,1)$ model, the $\operatorname{GM}(1,1)$ model is directly established using the data in the training set from 2012 to 2016, to forecast wind power generation from 2017Q1 to 2020Q1. And the predicted results are compared with actual data. Then, through using the MATLAB toolbox, the traditional $\operatorname{GM}(1,1)$ model can be directly established.

The parameters are $P=[a, b]^{T}=\left(B^{T} B\right)^{-1} B^{T} Y=[-0.0471,217.662]^{T}$.

Meanwhile, the corresponding time response function is $\hat{x}^{(1)}(k)=4825.274 e^{0.0471(k-1)}-4621.27$. Then, via the first order inverse accumulation generation operator (1-IAGO), we can get the restored value $\hat{x}^{(0)}(k)$. The predicted values from 2012Q1 to 2020Q1 are compared with the actual values, and the results are shown in Figure 7.

\subsubsection{Holt-winters model}

Exponential smoothing is an important method for time series prediction. However, simple exponential smoothing cannot properly remove the randomness of time series. As a result, more effective methods have been developed, such as the Holt-winter model [57]. This model was first proposed by Holt, and then Winters improved it, so it was called the Holt-Winters model. The model has a solid theoretical basis and a satisfactory expression of seasonality [19]. In general, there are two types of Holt-Winters models: the multiplication model and the addition model. The multiplication model is more popular, which is more accurate and robust than the addition form. 

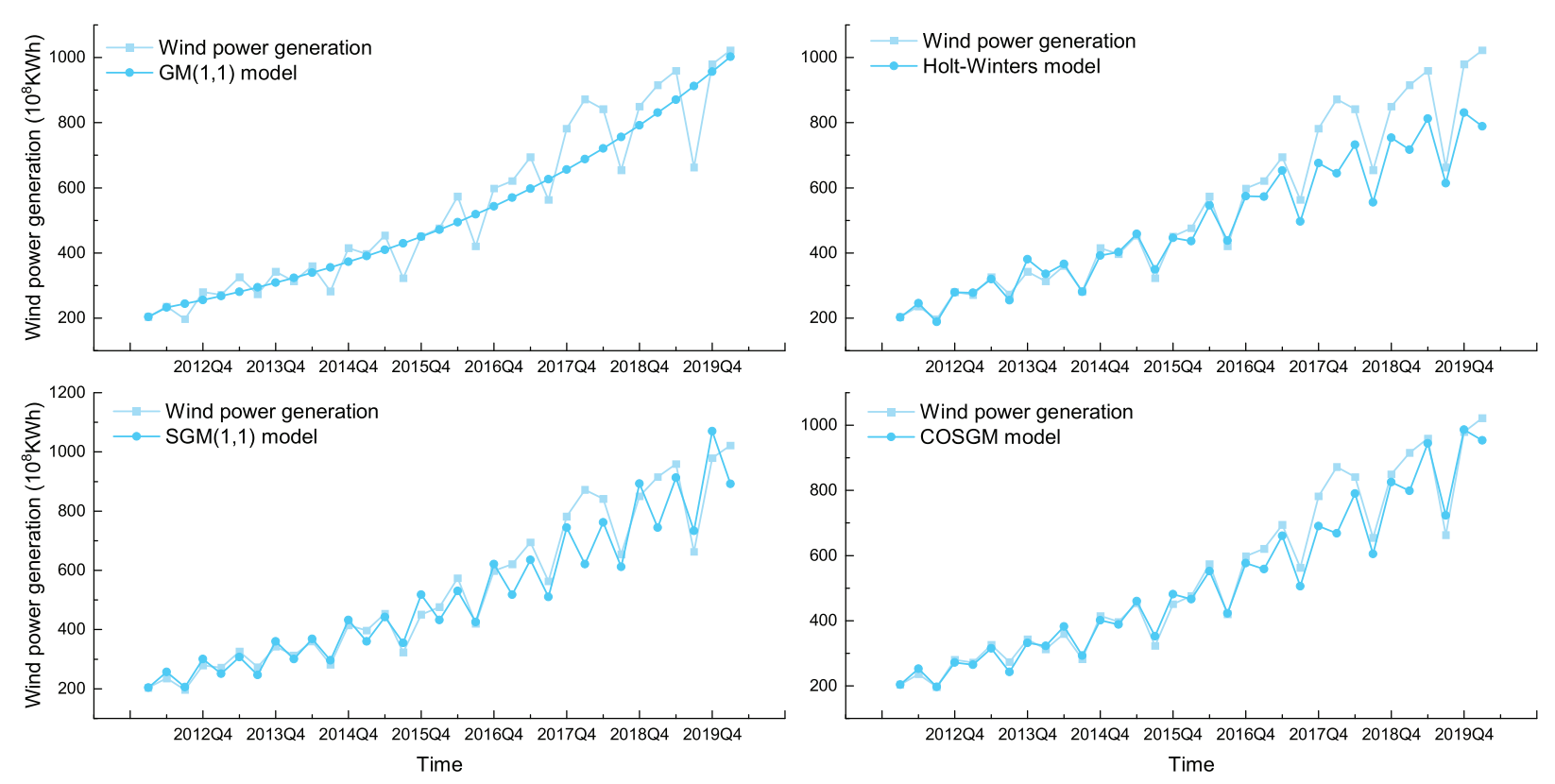

FIgURE 7 . The distributions of values predicted using the four models, and actual values: 2012Q1-2020Q1.

TABLE 2. The seasonal index of the Holt-Winters model in the training set.

\begin{tabular}{lllll}
\hline \hline & \multicolumn{4}{c}{ Seasonal index } \\
\cline { 2 - 5 } & $I_{1}$ & $I_{2}$ & $I_{3}$ & $I_{4}$ \\
\hline Value & 1.00 & 1.11 & 0.82 & 1.08 \\
\hline
\end{tabular}

Considering that the seasonal fluctuation increases gradually with the development of the trend, this paper used the multiplicative Holt-Winters model to model the wind power generation of the training set and to predict the wind power generation of the test set.

With the help of Eviews10, the Holt-Winters model can be established in this work. The prediction results based on the Holt-Winters model are shown in Figure 7, and Table 2 is the seasonal index estimated based on the Holt-Winters multiplication model.

\subsubsection{The seasonal $G M(1,1)$ model}

To enhance the forecasting ability of the grey forecast model for seasonal time series, Wang et al. introduced seasonal factors into the accumulating generation process. By changing the internal mechanism of the grey model, Wang established a grey prediction model based on the seasonal accumulation operator [51].

By referring to the modeling procedure of Wang et al., we can obtain the seasonal factors by using the corresponding period average method. The calculation results of the seasonal index are shown in Table 3 .

Then, the 1-SAGO is generated by seasonal factors and the $\operatorname{SGM}(1,1)$ model is established.

Where, $\hat{a}_{s}=\left[a_{s}, b_{s}\right]^{T}=[-0.0453,221.3892]^{T}$.

The corresponding time response function is $\hat{x}_{s}^{(2.1)}(k)=5107.917 e^{0.0453(k-1)}-4887.18$. From $\hat{x}_{s}^{(1)}(k)$, the prediction value $\hat{x}_{s}^{(0)}(k)$ can be obtained through 1-IAGO, and the results are shown in Figure 7 . 
TABLE 3 . The seasonal index of the $\operatorname{SGM}(1,1)$ model in the training set.

\begin{tabular}{lllll}
\hline \hline & \multicolumn{4}{c}{ Seasonal index } \\
\cline { 2 - 5 } & $I_{1}$ & $I_{2}$ & $I_{3}$ & $I_{4}$ \\
\hline Value & 0.92 & 1.08 & 0.83 & 1.16 \\
\hline
\end{tabular}

TABLE 4. The seasonal factors of the COSGM model in the training set.

\begin{tabular}{ccccc}
\hline \hline & \multicolumn{4}{c}{ Seasonal factors } \\
\cline { 2 - 5 } & $f(1)$ & $f(2)$ & $f(3)$ & $f(4)$ \\
\hline Value & 0.99 & 1.12 & 0.82 & 1.07 \\
\hline
\end{tabular}

\subsubsection{The COSGM model}

The modeling procedure of the COSGM model is as follows:

Firstly, based on the moving average filter algorithm, the trend sequence and seasonal sequence are obtained, and then the seasonal factors $f(s)$ is calculated. Since the data of the training set is quarterly data, the parameter used in the moving average filtering algorithm is $\theta_{k}=\{1,2,2,2,1\} / 8$. Under the assumption that the seasonal change is stable, we can calculate the seasonal factors in the training set, as shown in Table 4.

Secondly, The COSGM model can be constructed and the PSO algorithm can be used to search for the $r$-order of fractional order and initial value correction term $\varsigma$. Therefore, $r=0.9582$ and $\varsigma=0.7606$ can be obtained by using the PSO algorithm.

Third, the corresponding time response can be obtained by plugging $r$ and $\varsigma$ into the COSGM model. In the COSGM model, the parameters are $\hat{\beta}=\left[\hat{\beta}_{1}, \hat{\beta}_{2}\right]^{T}=[1.045,207.2996]^{T}$.

Finally, based on the seasonal factors, the predicted value can be obtained, and the results as shown in Figure 7.

\subsection{Comparison of prediction accuracy}

To better evaluate the prediction accuracy of the four models, RMSE, MAE, and MAPE are selected in this paper. And the calculation results are shown in Table 5. The performance of the four models in the test set are shown in Figure 8. In addition, to verify that the measurement of the seasonal effect of this model is more consistent with the actual situation, the COSGM model, SGM $(1,1)$ model, and Holt-Winters model are compared and analyzed in this paper to measure the seasonal effect of wind power generation. And the results are shown in Figure 9.

Overall, the traditional $\operatorname{GM}(1,1)$ model had the worst predictive effect in both the training set and the test set. The main reason why the prediction accuracy is not high is that the model cannot reflect the seasonal fluctuation characteristics. However, compared with the Holt-Winters model, the traditional GM(1,1) model has better mid-term and long-term forecasting ability due to its high mining ability for the long-term trend of a data sequence.

It can be seen from Table 5 and Figure 8 that the Holt-Winters model is significantly better than the GM(1,1) model and $\operatorname{SGM}(1,1)$ model in fitting the data sequences, and only worse than the $\operatorname{COSGM}(1,1)$ model. From Figure 7, although the Holt-Winters model can reflect the characteristics of seasonal fluctuation well, it is difficult to describe the long-term trend of the data series. This is mainly because the Holt-Winters model is suitable for linear trend and has weak adaptability to nonlinear trend. This is also the main reason that the prediction accuracy of the Holt-Winters model in the short-term and long-term predictions is worse than that 
TABLE 5. Comparison of prediction accuracy of the four models (2012Q1-2020Q1).

\begin{tabular}{|c|c|c|c|c|}
\hline & $\operatorname{GM}(1,1)$ & Holt-Winters & $\operatorname{SGM}(1,1)$ & COSGM \\
\hline \multicolumn{5}{|l|}{ Train set } \\
\hline $\operatorname{MAE}\left(10^{8} \mathrm{KWh}\right)$ & 35.90 & 14.62 & 22.56 & 13.51 \\
\hline RMSE $\left(10^{8} \mathrm{KWh}\right)$ & 48.12 & 18.85 & 27.34 & 16.46 \\
\hline MAPE (\%) & $10.16 \%$ & $3.97 \%$ & $6.17 \%$ & $3.80 \%$ \\
\hline \multicolumn{5}{|l|}{ Test set } \\
\hline MAE $\left(10^{8} \mathrm{KWh}\right)$ & 97.35 & 120.51 & 90.45 & 64.58 \\
\hline RMSE $\left(10^{8} \mathrm{KWh}\right)$ & 115.03 & 136.17 & 108.24 & 81.16 \\
\hline MAPE (2017Q1-2018Q4) (\%) & $13.42 \%$ & $13.06 \%$ & $11.14 \%$ & $9.58 \%$ \\
\hline MAPE (2019Q1-2020Q1) (\%) & $12.05 \%$ & $16.42 \%$ & $11.21 \%$ & $6.13 \%$ \\
\hline
\end{tabular}

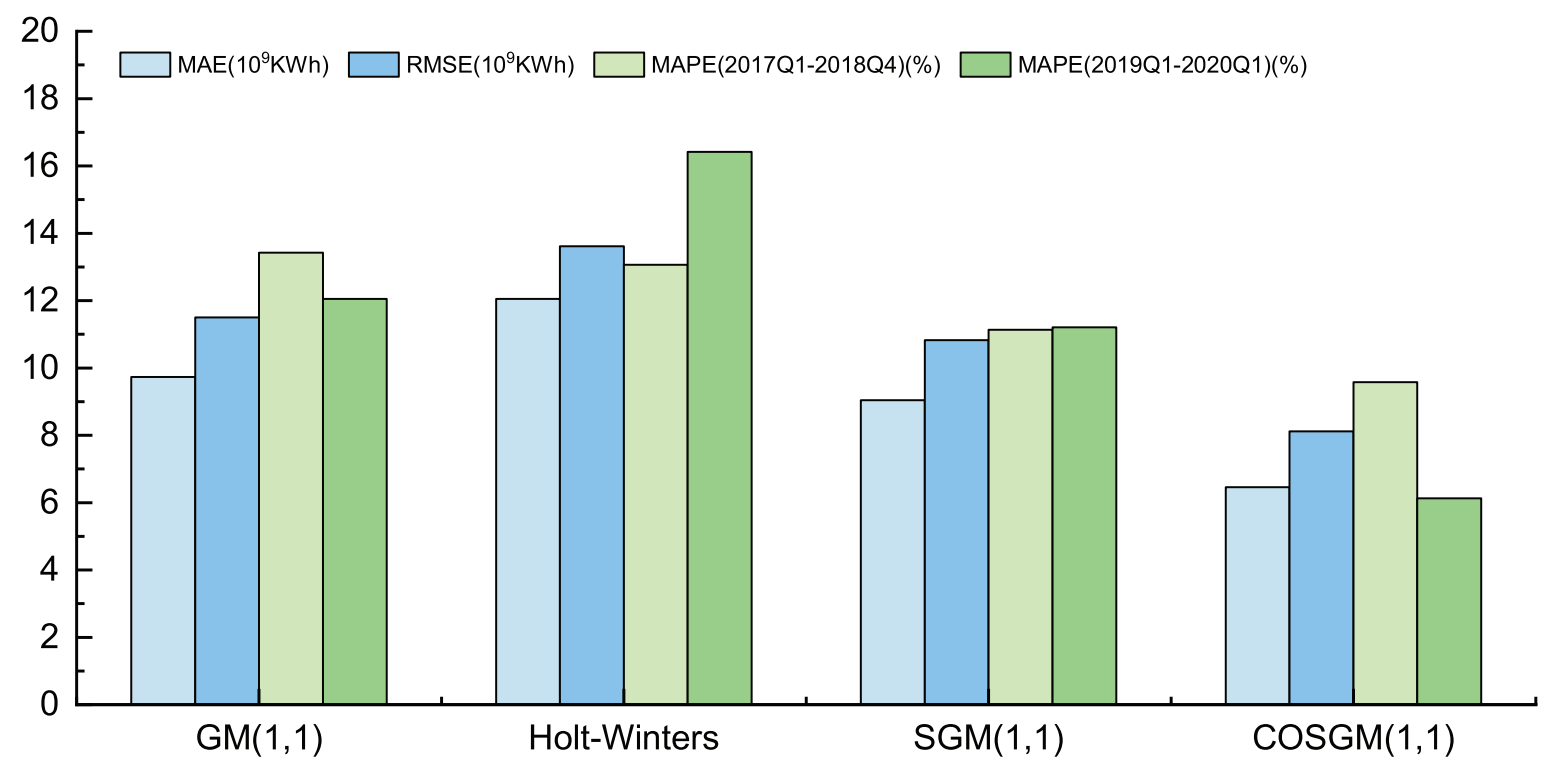

FiguRE 8. Comparison of testing errors.

of the $\operatorname{SGM}(1,1)$ model and the $\operatorname{COSGM}(1,1)$ model. When the long-term trend of the data series is linear, the Holt-Winters model has a satisfactory prediction effect.

The fitting effect of the $\operatorname{SGM}(1,1)$ model in the training set is only better than the traditional $\operatorname{GM}(1,1)$ model. Because the grey model well represents the long-term trend and the seasonal grey model considers the characteristics of seasonal fluctuations, the performance of the model in the test set is better than the HoltWinters model and the traditional $\operatorname{GM}(1,1)$. However, the prediction effect of the $\operatorname{SGM}(1,1)$ model is weaker than that of the COSGM model in both the training set and test set. From Figure 9, it can be seen that the $\operatorname{SGM}(1,1)$ model does not reflect the seasonal fluctuation characteristics well. This is mainly because the season factor of the $\operatorname{SGM}(1,1)$ model is a simple average method which is suitable for no obvious long-term trend [36], so it is difficult to accurately measure the seasonal effect of wind power generation. Holt-winter model and COSGM model measure the seasonal utility, in general, is consistent, the main reason for the difference is that the trend of the measure is not consistent. According to the effect of the training set, the COSGM model is reasonable in the measure of seasonality. 


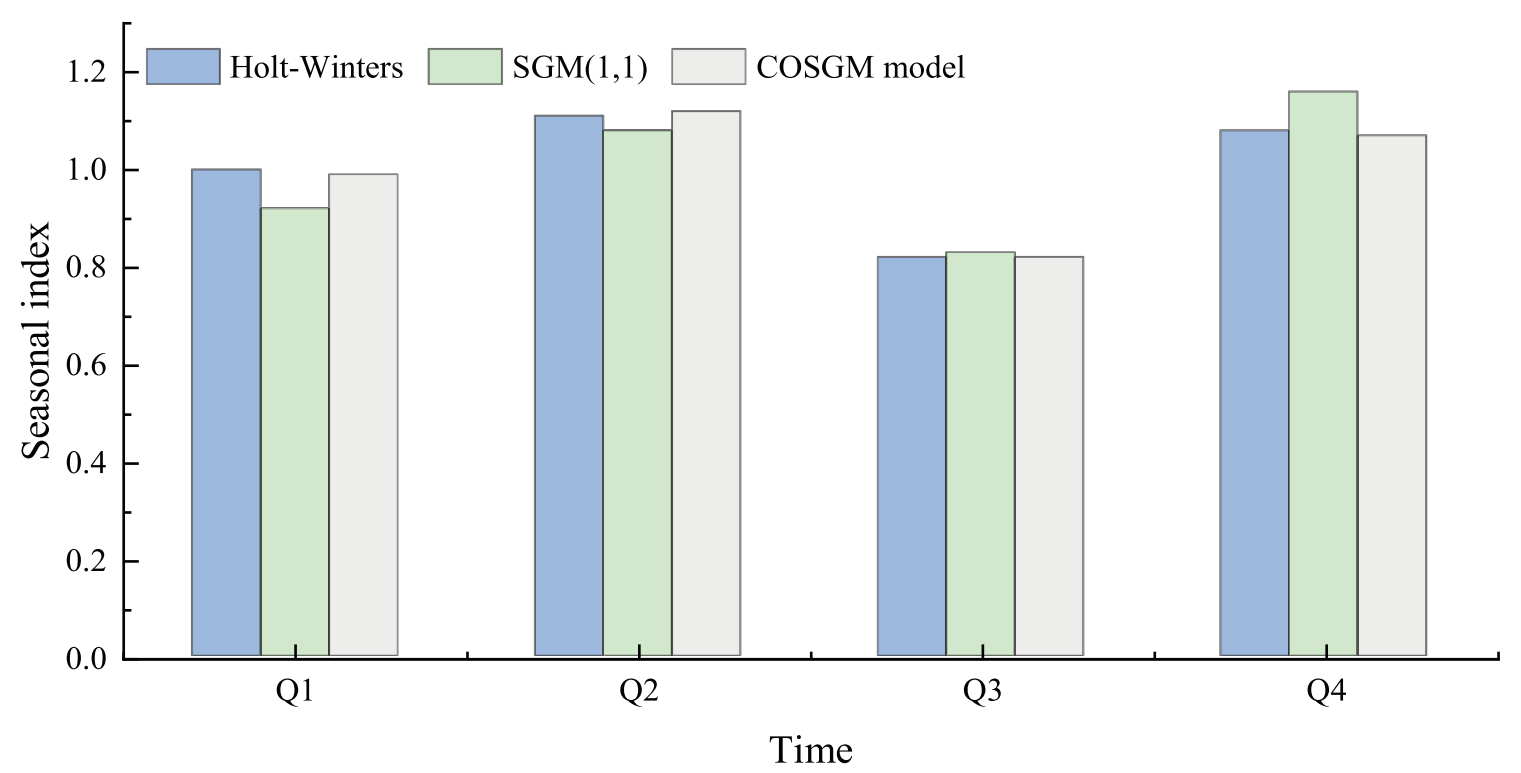

Figure 9. Comparison of the seasonal index of the three models.

Whether in the training set or the test set, the prediction effect of the COSGM model is better than the traditional GM(1,1) model, Holt-Winters model, and the $\operatorname{SGM}(1,1)$ model. There are three reasons for the high prediction accuracy of the COSGM model. Firstly, considering the seasonal characteristics of the data series, we can accurately measure the seasonality of the data series, so it has better seasonal characterization ability than $\operatorname{GM}(1,1)$ model and $\operatorname{SGM}(1,1)$ model. Secondly, we consider the long-memory property of the data series and the sensitivity of the initial value of the grey model, which is more in line with the criterion of new information first, so it has better tendency representation ability than other grey prediction models and Holt-Winters model. Thirdly, considering the trend and seasonality synthetically, the evolution law of the data series is dug more deeply, and it has better performance in the medium and long term. In general, it can be seen from Tables 1 and 5 that the COSGM model has a high prediction accuracy in both the short term and medium term. In the long run, with the stability of seasonal fluctuation, the prediction effect of this model will be better. Therefore, the COSGM model can provide important data support for future energy planning.

\subsection{Out of sample forecast of wind power generation in China from 2021Q2 to 2024Q2}

It can be seen from the analysis in Section 3.2 that the COSGM model, which comprehensively considers the characteristics of seasonal fluctuation and long-term trend, is suitable for modeling wind power generation in China. Therefore, in this section, COSGM is used to forecast wind power generation in China from 2021Q2 to 2024Q2. To make full use of the information and mine the long-term trend and seasonal fluctuation characteristics of the data series, this study established $\operatorname{COSGM}(1,1)$ model with all data from 2012Q1 to 2021Q1 to predict wind power generation in China from 2021Q2 to 2024Q2.

According to the modeling process of the COSGM model, we can calculate the seasonal factors, as shown in Table 6 .

Then, by using PSO, $r=0.9048$ and $\varsigma=0.7682$ can be obtained.

So, the corresponding time response can be obtained by plugging $r$ and $\varsigma$ into the COSGM model. In the COSGM model, the parameters are $\hat{\beta}=\left[\hat{\beta}_{1}, \hat{\beta}_{2}\right]^{T}=[1.0450,186.503]^{T}$. 
TABLE 6. The seasonal factors of the COSGM model (2012Q1-2021Q1).

\begin{tabular}{ccccc}
\hline \hline & \multicolumn{4}{c}{ Seasonal factors } \\
\cline { 2 - 5 } & $f(1)$ & $f(2)$ & $f(3)$ & $f(4)$ \\
\hline Value & 1.03 & 1.10 & 0.80 & 1.06 \\
\hline
\end{tabular}

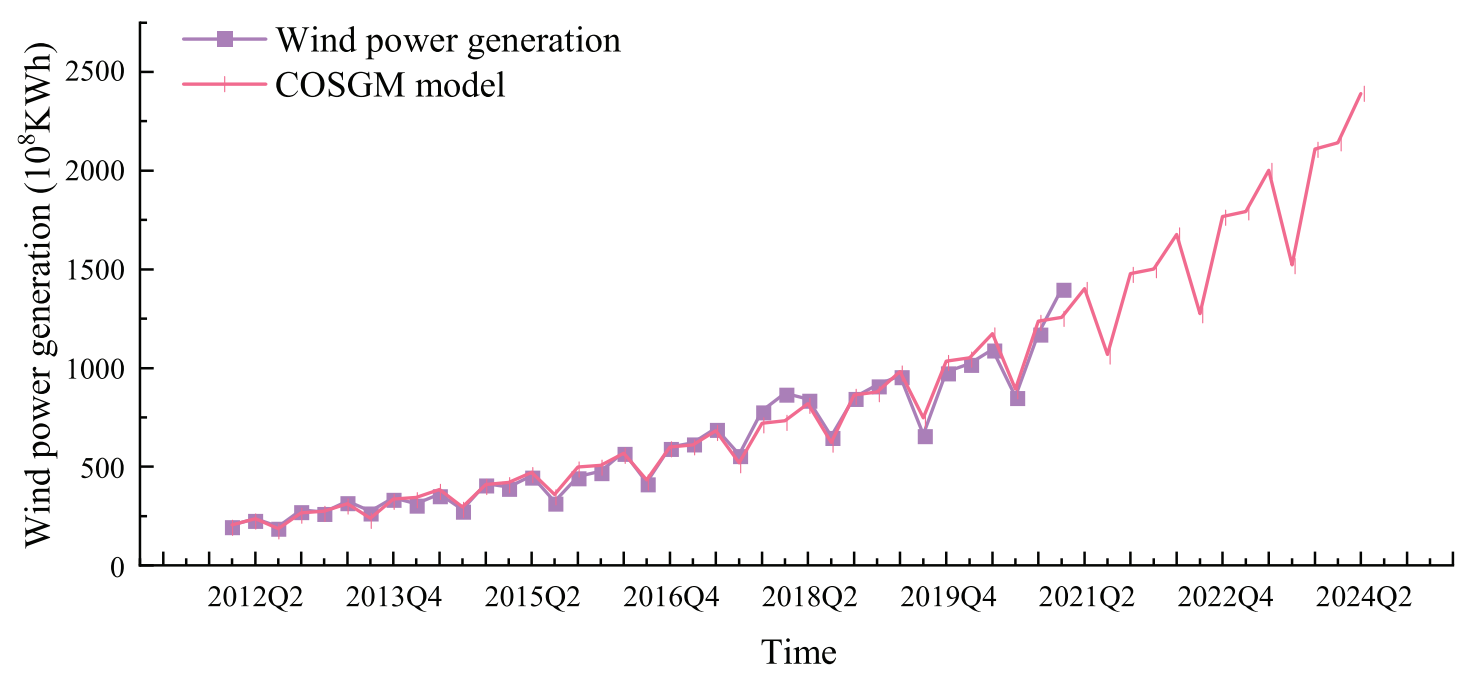

Figure 10. Comparison of values predicted by the COSGM model and the actual values: 2012Q1-2024Q2.

Finally, based on the seasonal factors, the predicted value can be obtained, and the results as shown in Figure 10. Table 7 shows the comparison between the fit values of the model and the actual values from 2012Q1 to 2021Q1.

During 2012Q1-2021Q1, the MAE, MAPE, and RMSE of the COSGM model are 32.66\%, 5.19\%, and 46.64\%, respectively. Therefore, COSGM has excellent prediction accuracy, and the prediction results of wind power generation in China from 2021Q2 to 2024Q2 are credible based on the MAPE. And the long-term forecasts are valid if the existing wind power expansion capacity policy is maintained in the next four years.

According to Figure 10, the total amount of wind power generation in China will continue to grow. It is estimated that by 2024Q2, the total amount of wind power will reach 239.09 TWh. Moreover, wind power generation in China presents a significant seasonal fluctuation characteristic. In the fourth quarter, wind power generation reached the highest, and in the third quarter, wind power generation reached the lowest.

\section{Conclusions And Discussions}

On the whole, starting from the seasonality of wind power generation, this paper proposes a new seasonal grey forecasting model based on collaborative optimization to excavate the development patterns of quarterly time series. And this proposed model accurately captures the seasonal characteristics and trend characteristics of the quarterly time series.

This proposed model is applied to forecast the wind power generation between 2012Q1 and 2020Q1 in China. And the prediction results are then compared with those obtained by using three existing approaches, $\operatorname{GM}(1,1)$ model, SGM $(1,1)$ model, and the Holt-Winters model. Comparative results indicate that the proposed method has higher prediction accuracy and is suitable for forecasting data series with seasonal fluctuations. 
TABLE 7. Fit values and actual values of COSGM model from 2012Q1 to 2021Q1.

\begin{tabular}{llll}
\hline \hline Time & Actual value & Forecasted value & Error (\%) \\
\hline 2012Q1 & 204.00 & 204.00 & $0.00 \%$ \\
2012Q2 & 235.70 & 235.70 & $0.00 \%$ \\
2012Q3 & 196.70 & 186.50 & $5.19 \%$ \\
2012Q4 & 279.80 & 265.30 & $5.18 \%$ \\
2013Q1 & 271.50 & 274.70 & $1.18 \%$ \\
2013Q2 & 325.70 & 311.20 & $4.45 \%$ \\
2013Q3 & 273.40 & 239.40 & $12.44 \%$ \\
2013Q4 & 342.50 & 334.90 & $2.22 \%$ \\
2014Q1 & 313.10 & 342.90 & $9.52 \%$ \\
2014Q2 & 360.00 & 385.50 & $7.08 \%$ \\
2014Q3 & 282.30 & 294.80 & $4.43 \%$ \\
2014Q4 & 414.90 & 410.40 & $1.08 \%$ \\
2015Q1 & 397.00 & 418.80 & $5.49 \%$ \\
2015Q2 & 453.80 & 469.40 & $3.44 \%$ \\
2015Q3 & 323.10 & 358.10 & $10.83 \%$ \\
2015Q4 & 450.80 & 497.50 & $10.36 \%$ \\
2016Q1 & 476.20 & 506.80 & $6.43 \%$ \\
2016Q2 & 573.80 & 567.10 & $1.17 \%$ \\
2016Q3 & 420.20 & 432.10 & $2.83 \%$ \\
2016Q4 & 598.10 & 599.70 & $0.27 \%$ \\
2017Q1 & 621.20 & 610.20 & $1.77 \%$ \\
2017Q2 & 694.50 & 682.30 & $1.76 \%$ \\
2017Q3 & 562.90 & 519.50 & $7.71 \%$ \\
2017Q4 & 782.10 & 720.50 & $7.88 \%$ \\
2018Q1 & 871.60 & 732.70 & $15.94 \%$ \\
2018Q2 & 841.60 & 818.80 & $2.71 \%$ \\
2018Q3 & 654.30 & 623.10 & $4.77 \%$ \\
2018Q4 & 850.10 & 863.80 & $1.61 \%$ \\
2019Q1 & 913.40 & 878.00 & $3.88 \%$ \\
2019Q2 & 959.40 & 980.90 & $2.24 \%$ \\
2019Q3 & 662.70 & 746.20 & $12.60 \%$ \\
2019Q4 & 979.20 & 1034.10 & $5.61 \%$ \\
2020Q1 & 1022.40 & 1050.90 & $2.79 \%$ \\
2020Q2 & 1094.60 & 1173.70 & $7.23 \%$ \\
2020Q3 & 854.90 & 892.60 & $4.41 \%$ \\
2020Q4 & 1174.10 & 1236.80 & $5.34 \%$ \\
2021Q1 & 1400.60 & 1256.60 & $10.28 \%$ \\
\hline & & &
\end{tabular}

Subsequently, we apply the proposed model to predict future wind power generation in China between 2021Q2 and 2024Q2. The results show that the long-term forecasts are valid if the existing wind power expansion capacity policy is maintained in the next four years. Our prediction confirms that wind power will continue to change seasonally, showing a steady upward trend. By 2024Q2, China's wind power generation is projected to reach 239.09 TWh. With the acceleration of China's construction of the energy industry, stable and sustained growth of wind power generation can effectively help optimize and upgrade the energy structure, and help realize the coordinated development of the economy and environment.

As demonstrated above, the COSGM model achieves a competitive performance in the quarterly wind power generation. The reason why the proposed model possesses high forecasting accuracy can be interpreted as below: 
(1) Compared with the other grey model, the fractional-order accumulation generation operator $(r$-AGO) is employed in the COSGM model to preprocess the wind power generation series. On the one hand, this technology significantly weakens the randomness of the data sequence and enhances the development trend of the data sequence. On the other hand, it also further excavates the hidden characteristics of the data sequence and adapts to the long memory characteristics of the data sequence. Moreover, the COSGM model is more in line with the criterion of new information priority and reduces the loss of information. And PSO algorithm is used to modify the initial value of the COSGM model, and further mining the potential information of the data sequence.

(2) In the proposed model, the moving average filtering algorithm is applied to realize the separation of the trend and season. Thus, compared with other grey prediction models, this paper measures the seasonal effect of wind power generation more accurately. This technology has a broad application prospect, it can not only adapt to various periodic characteristics but also reduce the interference of irregular fluctuations. However, as the symmetrical moving average filter will cause information loss, so this paper adopts the method of simultaneous optimization of trend and season to compensate for the loss of information.

However, there are still many new research opportunities that are worth exploring in the future. On the one hand, the COSGM model divides the original sequence into a trend and a periodic component, and regards the seasonal fluctuation as the pure influence of seasonal factors on the original trend, but ignores the role of irregular factors. How to further analyze and predict the role of irregular factors will be a valuable topic in the future.

On the other hand, the proposed model is based on the premise that the seasonal effect of the original sequence is stable. If the seasonal effect of the original sequence develops and changes over time, the model will be difficult to measure the seasonal effect. Therefore, the future work is how to further capture the dynamic changes of seasonal effects and adapt to the development of seasonal patterns.

Acknowledgements. This work is partially funded by the Humanities and Social Science Foundation of the Ministry of Education (18YJA630088) and the Fundamental Research Funds for the Central Universities (2019JDZD06). Even so, the work does not involve any conflict of interest.

\section{REFERENCES}

[1] O. Abedinia, M. Lotfi, M. Bagheri, B. Sobhani, M. Shafie-khah and J.P.S. Catalão, Improved EMD-based complex prediction model for wind power forecasting. IEEE Trans. Sustain. Energy 11 (2020) 2790-802.

[2] R. Abhinav, N.M. Pindoriya, J. Wu and C. Long, Short-term wind power forecasting using wavelet-based neural network. Energy Proc. 142 (2017) 455-460.

[3] R. Azimi, M. Ghofrani and M. Ghayekhloo, A hybrid wind power forecasting model based on data mining and wavelets analysis. Energy Convers. Manage. 127 (2016) 208-225.

[4] D. Baptista, J.P. Carvalho and F. Morgado-Dias, Comparing different solutions for forecasting the energy production of a wind farm. Neural Comput. Appl. 32 (2020) 15825-15833.

[5] H.H. Çevik, M. Çunkaş and K. Polat, A new multistage short-term wind power forecast model using decomposition and artificial intelligence methods. Phys. Stat. Mech. Appl. 534 (2019) 122177.

[6] J. Dai, Y. Tan and X. Shen, Investigation of energy output in mountain wind farm using multiple-units SCADA data. Appl. Energy 239 (2019) 225-38.

[7] H. Demolli, A.S. Dokuz, A. Ecemis and M. Gokcek, Wind power forecasting based on daily wind speed data using machine learning algorithms. Energy Convers. Manage. 198 (2019) 111823.

[8] H.S. Dhiman, D. Deb and J.M. Guerrero, Hybrid machine intelligent SVR variants for wind forecasting and ramp events. Renew. Sustain. Energy Rev. 108 (2019) 369-379.

[9] S. Ding, R. Li and Z. Tao, A novel adaptive discrete grey model with time-varying parameters for long-term photovoltaic power generation forecasting. Energy Convers. Manage. 227 (2021) 113644.

[10] E. Erdem and J. Shi, ARMA based approaches for forecasting the tuple of wind speed and direction. Appl. Energy 88 (2011) 1405-1414.

[11] A.A. Ezzat, Turbine-specific short-term wind speed forecasting considering within-farm wind field dependencies and fluctuations. Appl. Energy 269 (2020) 115034.

[12] A. Fabbri, T.G. San Román, J.R. Abbad and V.H.M. Méndez Quezada, Assessment of the cost associated with wind generation prediction errors in a liberalized electricity market. IEEE Trans. Power Syst. 20 (2005) 1440-1446. 
[13] S.J. Ghoushchi, S. Manjili, A. Mardani and M.K. Saraji, An extended new approach for forecasting short-term wind power using modified fuzzy wavelet neural network: a case study in wind power plant. Energy 223 (2021) 120052.

[14] S. Guefano, J.G. Tamba, T.E.W. Azong and L. Monkam, Methodology for forecasting electricity consumption by Grey and Vector autoregressive models. MethodsX 8 (2021) 101296.

[15] Z. Guo, W. Zhao, H. Lu and J. Wang, Multi-step forecasting for wind speed using a modified EMD-based artificial neural network model. Renew. Energy 37 (2012) 241-249.

[16] K. Higashiyama, Y. Fujimoto and Y. Hayashi, Feature extraction of NWP data for wind power forecasting using 3Dconvolutional neural networks. Energy Proc. 155 (2018) 350-358.

[17] R.J. Hodrick and E.C. Prescott, Postwar U.S. business cycles: an empirical investigation. J Money, Credit Bank 29 (1997) 1.

[18] J. Hu, J. Wang and G. Zeng, A hybrid forecasting approach applied to wind speed time series. Renew. Energy 60 (2013) $185-194$.

[19] R.J. Hyndman, A.B. Koehler, R.D. Snyder and S. Grose, A state space framework for automatic forecasting using exponential smoothing methods. Int. J. Forecast. 18 (2002) 439-454.

[20] W.D. Jacondino, A.L. da Silva Nascimento, L. Calvetti, G. Fisch, C.A.A. Beneti and S.R. da Paz, Hourly day-ahead wind power forecasting at two wind farms in northeast Brazil using WRF model. Energy 230 (2021) 120841.

[21] S.M.J. Jalali, S. Ahmadian, M. Khodayar, A. Khosravi, V. Ghasemi, M. Shafie-khah, S. Nahavandi and J.P. Catalão, Towards novel deep neuroevolution models: chaotic levy grasshopper optimization for short-term wind speed forecasting. Eng. Comput. (2021) DOI: $10.1007 / \mathrm{s} 00366-021-01356-0$.

[22] S.A. Javed, B. Zhu and S. Liu, Forecast of biofuel production and consumption in top $\mathrm{CO}_{2}$ emitting countries using a novel grey model. J. Clean. Prod. 276 (2020) 123997.

[23] Z.Q. Jia, Z.F. Zhou, H.J. Zhang, B. Li and Y.X. Zhang, Forecast of coal consumption in Gansu Province based on Grey-Markov chain model. Energy 199 (2020) 117444.

[24] W. Jiang, X. Wu, Y. Gong, W. Yu and X. Zhong, Holt-Winters smoothing enhanced by fruit fly optimization algorithm to forecast monthly electricity consumption. Energy 193 (2020) 807-814.

[25] D. Ju-Long, Control problems of grey systems. Syst. Control Lett. 1 (1982) 288-294.

[26] R.G. Kavasseri and K. Seetharaman, Day-ahead wind speed forecasting using f-ARIMA models. Renew Energy 34 (2009) $1388-1393$.

[27] A. Kisvari, Z. Lin and X. Liu, Wind power forecasting - a data-driven method along with gated recurrent neural network. Renew. Energy 163 (2021) 1895-909.

[28] A. Kusiak and W. Li, Short-term prediction of wind power with a clustering approach. Renew. Energy 35 (2010) $2362-2369$.

[29] L. Lazić, G. Pejanović and M. Živković, Wind forecasts for wind power generation using the ETA model. Renew Energy 35 (2010) 1236-1243.

[30] C.D. Lewis, A Radical Guide to Exponential Smoothing and Curve Fitting. Butterworth-Heinemann (1982).

[31] S. Li, Y. Miao, G. Li and M. Ikram, A novel varistructure grey forecasting model with speed adaptation and its application. Math. Comput. Simul. 172 (2020) 45-70.

[32] N. Li, J. Wang, L. Wu and Y. Bentley, Predicting monthly natural gas production in China using a novel grey seasonal model with particle swarm optimization. Energy 215 (2021) 119118.

[33] W. Lifeng, L. Sifeng and Y. Li-gen, Discrete grey model based on fractional order accumulate. Syst. Eng. Theory Pract. 34 (2014) 1822-1827.

[34] Y.-H. Lin and P.-C. Lee, Novel high-precision grey forecasting model. Autom. Constr. 16 (2007) 771-777.

[35] Z. Lin and X. Liu, Wind power forecasting of an offshore wind turbine based on high-frequency SCADA data and deep learning neural network. Energy 201 (2020) 117693.

[36] F. Liu and H.G. Zhang, Research status and existing problems of seasonal index. Stat. Decis. (2008) 11-14.

[37] X. Liu, B. Moreno and A.S. García, A grey neural network and input-output combined forecasting model. Primary energy consumption forecasts in Spanish economic sectors. Energy 115 (2016) 1042-1054.

[38] X. Ma, X. Mei, W. Wu, X. Wu and B. Zeng, A novel fractional time delayed grey model with Grey Wolf Optimizer and its applications in forecasting the natural gas and coal consumption in Chongqing China. Energy 178 (2019) $487-507$.

[39] J.W. Messner and P. Pinson, Online adaptive lasso estimation in vector autoregressive models for high dimensional wind power forecasting. Int. J. Forecast. 35 (2019) 1485-1498.

[40] N. Mohan, K.P. Soman and S. Sachin Kumar, A data-driven strategy for short-term electric load forecasting using dynamic mode decomposition model. Appl. Energy 232 (2018) 229-244.

[41] S. Moonchai and N. Chutsagulprom, Short-term forecasting of renewable energy consumption: augmentation of a modified grey model with a Kalman filter. Appl. Soft Comput. 87 (2020) 105994.

[42] J. Naik, R. Bisoi and P.K. Dash, Prediction interval forecasting of wind speed and wind power using modes decomposition based low rank multi-kernel ridge regression. Renew. Energy 129 (2018) 357-383.

[43] N. Norouzi and M. Fani, Black gold falls, black plague arise - an Opec crude oil price forecast using a gray prediction model. Upstream Oil Gas Technol. 5 (2020) 100015.

[44] D. Prieto-Herráez, L. Frías-Paredes, J.M. Cascón, S. Lagüela-López, M. Gastón-Romeo, M.I. Asensio-Sevilla, I. Martín-Nieto, P.M. Fernandes-Correia, P. Laiz-Alonso, O.F. Carrasco-Díaz and C. Saez-Blazquez, Local wind speed forecasting based on WRF-HDWind coupling. Atmos. Res. 248 (2021) 105219.

[45] M.A. Prósper, C. Otero-Casal, F.C. Fernández and G. Miguez-Macho, Wind power forecasting for a real onshore wind farm on complex terrain using WRF high resolution simulations. Renew. Energy 135 (2019) 674-686. 
[46] W.Y. Qian and Y.G. Dang, GM(1, 1) model based on oscillation sequences. Syst. Eng. Pract. 29 (2009) $149-154$.

[47] W. Qian and J. Wang, An improved seasonal GM(1,1) model based on the HP filter for forecasting wind power generation in China. Energy 209 (2020) 118499.

[48] U. Şahin, Future of renewable energy consumption in France, Germany, Italy, Spain, Turkey and UK by 2030 using optimized fractional nonlinear grey Bernoulli model. Sustain. Prod. Consum. 25 (2021) 1-14.

[49] J. Wang, X. Ma, J. Wu and Y. Dong, Optimization models based on GM $(1,1)$ and seasonal fluctuation for electricity demand forecasting. Int. J. Electr. Power Energy Syst. 43 (2012) 109-117.

[50] Z.X. Wang, Q. Li and L.L. Pei, Grey forecasting method of quarterly hydropower production in China based on a data grouping approach. Appl. Math. Model. 51 (2017) 302-316.

[51] Z.X. Wang, Q. Li and L.L. Pei, A seasonal GM(1,1) model for forecasting the electricity consumption of the primary economic sectors. Energy 154 (2018) 522-534.

[52] Z.X. Wang, L.Y. He and H.H. Zheng, Forecasting the residential solar energy consumption of the United States. Energy $\mathbf{1 7 8}$ (2019) 610-623.

[53] Z.X. Wang, Z.W. Wang and Q. Li, Forecasting the industrial solar energy consumption using a novel seasonal GM(1,1) model with dynamic seasonal adjustment factors. Energy 200 (2020) 117460.

[54] G. Wang, R. Jia, J. Liu and H. Zhang, A hybrid wind power forecasting approach based on Bayesian model averaging and ensemble learning. Renew. Energy 145 (2020) 2426-2434.

[55] C. Wang, H. Zhang and P. Ma, Wind power forecasting based on singular spectrum analysis and a new hybrid Laguerre neural network. Appl. Energy 259 (2020) 114139.

[56] R. Weron, Measuring long-range dependence in electricity prices BT, edited by H. Takayasu. In: Empirical Science of Financial Fluctuations. Springer Japan, Tokyo (2002) 110-119.

[57] P.R. Winters, Forecasting sales by exponentially weighted moving averages. Manage. Sci. 6 (1960) 324-342.

[58] L. Wu, S. Liu, L. Yao, S. Yan and D. Liu, Grey system model with the fractional order accumulation. Commun. Nonlinear Sci. Numer. Simul. 18 (2013) 1775-1785.

[59] W. Wu, X. Ma, B. Zeng, Y. Wang and W. Cai, Forecasting short-term renewable energy consumption of China using a novel fractional nonlinear grey Bernoulli model. Renew. Energy 140 (2019) 70-87.

[60] W.-Z. Wu, H. Pang, C. Zheng, W. Xie and C. Liu, Predictive analysis of quarterly electricity consumption via a novel seasonal fractional nonhomogeneous discrete grey model: a case of Hubei in China. Energy 229 (2021) 120714.

[61] X. Xiao, J. Yang, S. Mao and J. Wen, An improved seasonal rolling grey forecasting model using a cycle truncation accumulated generating operation for traffic flow. Appl. Math. Model. 51 (2017) 386-404.

[62] N.M. Xie and S.F. Liu, Discrete grey forecasting model and its optimization. Appl. Math. Model. 33 (2009) $1173-1186$.

[63] C. Yildiz, H. Acikgoz, D. Korkmaz and U. Budak, An improved residual-based convolutional neural network for very short-term wind power forecasting. Energy Convers. Manage. 228 (2021) 113731.

[64] M.U. Yousuf, I. Al-Bahadly and E. Avci, A modified GM(1,1) model to accurately predict wind speed. Sustain. Energy Technol. Assess. 43 (2021) 100905.

[65] Y. Zhang, A Study of the Theory and Application of Structural Time Series Model in Seasonal Adjustment (in Chinese). Nankai University (2013).

[66] S. Zhou, Y. Wang, Y. Zhou, L.E. Clarke and J.A. Edmonds, Roles of wind and solar energy in China's power sector: implications of intermittency constraints. Appl. Energy 213 (2018) 22-30.

[67] W. Zhou, X. Wu, S. Ding and J. Pan, Application of a novel discrete grey model for forecasting natural gas consumption: a case study of Jiangsu Province in China. Energy 200 (2020) 117443.

\section{Subscribe to Open (S2O) A fair and sustainable open access model}

This journal is currently published in open access under a Subscribe-to-Open model (S2O). S2O is a transformative model that aims to move subscription journals to open access. Open access is the free, immediate, online availability of research articles combined with the rights to use these articles fully in the digital environment. We are thankful to our subscribers and sponsors for making it possible to publish this journal in open access, free of charge for authors.

\section{Please help to maintain this journal in open access!}

Check that your library subscribes to the journal, or make a personal donation to the $\mathrm{S} 2 \mathrm{O}$ programme, by contacting subscribers@edpsciences.org

More information, including a list of sponsors and a financial transparency report, available at: https://www. edpsciences.org/en/maths-s2o-programme 